\title{
Comprehensive transcriptional profiling and mouse phenotyping reveals dispensable role for adipose tissue selective long noncoding RNA Gm15551
}

Christoph Andreas Engelhard ${ }^{1}$, Chien Huang ${ }^{1,2}$, Sajjad Khani ${ }^{3,4}$, Petr Kasparek ${ }^{5}$, Jan Prochazka ${ }^{5}$, Jan Rozman ${ }^{5}$, David Pajuelo Reguera ${ }^{5}$, Radislav Sedlacek ${ }^{5}$ and Jan-Wilhelm Kornfeld ${ }^{1,3}{ }^{*}$

${ }^{1}$ Department for Biochemistry and Molecular Biology (BMB), University of Southern Denmark, Campusvej 55, 5230 Odense M, Denmark. ${ }^{2}$ Laboratory of Animal Physiology, Department of Animal Science and Technology, National Taiwan University, Taipei, 10617, Taiwan. ${ }^{3}$ Max Planck Institute for Metabolism Research, Gleueler Strasse 50, 50931 Köln, Germany. ${ }^{4}$ Institute for Diabetes and Cancer (IDC); Helmholtz Zentrum München, German Research Center for Environmental Health, Neuherberg, Germany. ${ }^{5}$ Czech Centre for Phenogenomics, Prumyslova 595, 25250 Vestec, Czech Republic. * Corresponding author. e-mail: janwilhelmkornfeld@bmb.sdu.dk

\section{Abstract}

Cold and nutrient activated brown adipose tissue (BAT) is capable of increasing systemic energy expenditure via uncoupled respiration and secretion of endocrine factors thereby protecting mice against diet-induced obesity and improving insulin response and glucose tolerance in men. Long non-coding RNAs (lncRNAs) have recently been identified as fine tuning regulators of cellular function. While certain lncRNAs have been functionally characterised in adipose tissue, their overall contribution in the activation of BAT remains elusive. We identified lncRNAs correlating to interscapular brown adipose tissue (iBAT) function in high fat diet (HFD) and cold stressed mice. We focused on Gm15551 which has an adipose tissue specific expression profile, is highly upregulated during adipogenesis and downregulated by $\beta$-adrenergic activation in mature adipocytes. Albeit we performed comprehensive transcriptional and adipocyte physiology profiling in vitro and in vivo, we could not detect an effect of gain or loss of function of Gm15551.

\section{Key points}

long noncoding RNAs; brown adipocytes

\section{Abbreviations}

ANCOVA analysis of covariances 
ANOVA analysis of variances

ATP adenosine triphosphate

BAT brown adipose tissue

cDNA complementary DNA

ChIP chromatin immunoprecipitation

DMEM Dulbecco's modified Eagle's medium

DNA deoxyribonucleic acid

eRNA enhancer RNA

eWAT epididymal white adipose tissue

FBS fetal bovin serum

GO gene ontology

HFD high fat diet

iBAT interscapular brown adipose tissue

IBMX 3-isobutyl-1-methylxanthin

IPGTT intraperitoneal glucose tolerance test

iWAT inguinal white adipose tissue

LNA locked nucleic acid

$\log$ FC $\log 2$ fold change

lncRNA long non-coding RNA

LRT likelihood ratio test

miRNA micro RNA

qPCR quantitative polymerase chain reaction

RNA ribonucleic acid

SDS Sodium dodecyl sulfate

sgRNA single guide RNA

siRNA small interfering RNA

T3 triiodothyronine

TRAP translating ribosomal affinity purification

WAT white adipose tissue

\section{Introduction}

The prevalence of obesity is increasing worldwide (NCD Risk Factor Collaboration (NCD-RisC), 2016). Obesity is the result of a chronic imbalance between energy intake and expenditure resulting in the accumulation of excess adipose tissue. Obesity is correlated with increased overall mortality and is a risk factor for various diseases including cardio vascular disease and diabetes type 2 (Angelantonio et al., 2016; Prospective Studies Collaboration, 2009).

Adipose tissue plays a central role in the regulation of energy balance. While white adipose tissue (WAT) mainly functions as storage of excess energy in the form of triglycerides, BAT is a highly metabolically active tissue (Rosen and Spiegelman, 2014). Morphologically, BAT is densely packed 
with mitochondria and generates heat by short-circuiting the mitochondrial proton gradient via uncoupling protein 1 (UCP1), facilitating substrate use without ATP generation (Cannon and Nedergaard, 2004). Thereby active BAT significantly improves glucose and lipid clearance and raises energy expenditure (Betz and Enerbäck, 2017; Klepac et al., 2019). Additionally, active BAT signals to other tissues improving the whole body metabolic profile via the secretion of endocrine factors and micro RNA (miRNA) containing exosomes (Scheele and Wolfrum, 2020; Zhang et al., 2019). In this regard, the recent demonstration of the presence of active BAT in adult humans has led to an increased interest in understanding the molecular signals underlying BAT differentiation and function (Betz and Enerbäck, 2017; Nedergaard et al., 2007).

The characterisation of the human transcriptome in the course of the ENCODE project revealed pervasive transcription of three quarters of the human genome (Djebali et al., 2012). Most of the transcribed sequences however do not fall within protein coding regions but give rise to non-coding ribonucleic acid (RNA) such as lncRNA (Djebali et al., 2012). lncRNAs are defined as non-coding genes giving rise to transcripts of more than $200 \mathrm{nt}$, which do not belong to an otherwise functionally defined class of RNA (Gil and Ulitsky, 2019). The lack of a functional definition coincides with a broad range of modes of function: lncRNAs have been shown to act both in cis as well as in trans (Gil and Ulitsky, 2019; Yao et al., 2019) via an interaction of the transcribed RNA molecule with other RNA, proteins or the DNA (Nguyen et al., 2018; Yi et al., 2020). Compared to coding genes, IncRNAs are on average lower expressed but show more tissue and developmental stage specific expression profiles, advocating for a role as fine tuning regulators of cellular function (Derrien et al., 2012). Selected lncRNAs have been shown to interfere with adipose tissue function and differentiation such as IncBATE10 which acts as a decoy for Celf1 which would otherwise bind to and repress Pgcla mRNA (Bai et al., 2017), H19 which functions as a BAT-specific gatekeeper of paternally expressed genes (Schmidt et al., 2018) and Ctcflos which regulated expression and splicing of Prdm16 (BastHabersbrunner et al., 2021). However, their overall contribution to these processes remains elusive.

In this study, we performed RNA sequencing on BAT from C57BL/6 mice challenged with coldtreatment and high-fat diet, two physiologically relevant models of BAT activation (Alcalá et al.,2017; Cannon and Nedergaard, 2004) as well as on a set of seven metabolically active tissues. We found a set of adipose tissue specific cold and/or diet regulated lncRNAs, from which we selected Gm15551 as a candidate for functional studies. The genomic locus of Gm15551 is bound by Pparg and Prdm16 in brown adipocytes and it is upregulated in adipogenesis, and downregulated upon $\beta$-adrenergic stimulation in adipocytes. We performed comprehensive phenotyping of gain and loss of function in vitro as well as loss of function in vivo but could not detect any phenotype related to Gm15551.

\section{Results}

\section{Total RNA-seq identifies IncRNAs regulated in activated iBAT}

In order to identify lncRNAs implicated in the regulation of iBAT function we set out to perform total RNA-seq on C57BL/6N mice put on a high fat diet regime from 8 weeks of age onwards for 12 weeks and additionally housed at $4{ }^{\circ} \mathrm{C}$ for $24 \mathrm{~h}$ at the end of this period (Fig $1 \mathrm{~A}$ ). We found that cold treatment significantly reduced the weight of the iBAT in both the control and the HFD group (Fig S1A), while HFD treatment alone did not induce significant changes in iBAT weight. On the other hand, epididymal white adipose tissue (eWAT) and inguinal white adipose tissue (iWAT) as well as liver weights were increased upon HFD treatment, independent of the cold treatment. Cold treatment 
alone only induced an increase of eWAT but not iWAT and liver weight. Gene expression measurements reflected the observed difference in the reaction of white and brown adipose tissue to the treatments (Fig S1B). Cold treatment induced a robust induction of the common adipose marker gene Elovl3 as well as the brown adipose markers Cidea, Dio2 and Ucp1 while HFD alone was insufficient for the induction of any significant changes in those genes, although $U c p 1$ was tendentiously upregulated. In iWAT however, cold and HFD treatment showed opposing effects. The white adipose marker gene Lep was tendentiously repressed upon cold treatment and induced by HFD while Elovl3, Dio2 and Ucp1 were upregulated by the cold and downregulated by the HFD treatment. The treatment regimes also directly affected the animals' metabolism as seen by the significantly impaired glucose tolerance upon HFD treatment (Fig $[\mathrm{S1}$ ). Energy expenditure was elevated by cold treatment but reduced by HFD treatment (Fig S1D) while the respiratory exchange rates indicated a shift towards lipid catabolism induced by both cold and HFD treatment (data not shown). Together, these data indicate our dataset is an adequate model for different functional states of iBAT. Additionally, we used a second dataset consisting of seven metabolically active tissues (iBAT, iWAT, eWAT, liver, kidney, muscle, heart) which we have generated for a previous study to be able to assess transcriptome wide tissue specificity (Pradas-Juni et al., 2020). To generate a comprehensive set of lncRNA genes, we combined the annotated transcript isoforms from GENCODE and the lncRNA isoforms from RNAcentral on gene level.

Total RNA-seq of the iBAT dataset identified 2490 differentially expressed genes, among them 216 lncRNA genes (likelihood ratio test (LRT), $p<0.001$; Fig 1B). The largest cluster (cluster 3) consisted of genes that were induced by cold treatment independent of the diet and was enriched for genes involved in stress response and mitochondrion organisation (Fig 1 $\mathrm{C}$, Fig $[\mathrm{S1E}$ ). Similarly, cluster 4 contained genes downregulated by cold treatment independently of diet and was enriched for gene expression regulation and signal transduction. The other clusters included genes with synergistic interaction of HFD and housing temperature; either being induced (cluster 5, enriched for signalling) or repressed by HFD and cold treatment (clusters 1 and 2, enriched for extracellular matrix as well as metabolism). We used the transcriptomics data from the seven metabolically active tissues to calculate an adipose tissue enrichment score, defined as the ratio of log adipose tissue counts over the log of total counts. As temperature influenced the gene expression of more genes then HFD, we focused on genes regulated by the cold treatment. Wald tests identified 110 cold regulated lncRNA genes $(s<0.05)$, of which $65(59 \%)$ also showed an adipose tissue specific expression profile (adipose score $>50 \%$; Fig 1D, Fig S1G). On the other hand, from the 610 cold regulated coding genes, only $35 \%$ showed adipose tissue specific expression (Fig S1F). Noteworthy, our analysis identified known brown adipose marker genes such as $U c p 1$ and Adcy3 as well as the lncRNA genes LncBate10 and Ctcflos, which have previously been shown to play a role in regulation of brown/beige adipose tissue function (Bai et al., 2017; Bast-Habersbrunner et al., 2021), proving the applicability of our strategy towards the identification of novel candidate adipose regulating lncRNAs. In order to rule out that any of the identified lncRNA genes were differentially regulated because of an increased immune cell infiltration of the iBAT caused by the cold or HFD treatment (Alcalá et al.,2017), we checked the expression profiles of several immune cell marker genes (Henriques et al., 2020), of which none were differentially regulated $(\mathrm{Fig} \mathrm{S} 1 \mathrm{H})$. 


\section{Gm15551 is an adipose specific, highly regulated IncRNA}

We further focused our study on the lncRNA Gm15551, which was highly adipose specific and significantly repressed upon cold treatment in iBAT (Fig[1D). While HFD alone was not sufficient to induce the repression of Gm15551 expression, the combination of cold treatment and HFD further repressed Gm15551 compared to cold treatment alone (ANOVA; $p=0.000701$, Fig 2A). Among the examined tissues, the expression of Gm15551 was observed to be strictly restricted to adipose tissue, similar to the common adipocyte marker genes Adipoq and Pparg (Fig 23). Within the three adipose tissues we looked at, Gm15551 showed the highest expression in eWAT and the lowest in iBAT, with intermediate expression in iWAT, anti-correlating to the expression of the thermogenic adipocyte marker gene Cidea. The notion of this expression pattern together with the repression upon activation of thermogenesis in iBAT led us to hypothesize Gm1555 might have an anti-thermogenic function.

Gm15551 is expressed from chromosome 3 and there are 2 transcripts annotated which both consist of 2 exons and only differ in the exact position of the transcription end site (Fig $2 \mathrm{C}$ ). Analysis of publicly available ChIP-Seq data showed that the locus is bound by the core thermogenic transcription factor Prdm16 in iBAT. Additionally, we found that Pparg binds to the Gm15551 locus in eWAT, iWAT as well as iBAT with the height of the ChIP-Seq peak correlating with the Gm15551 RNA expression levels. Chromatin features such as the ratio of $\mathrm{H} 3 \mathrm{~K} 4 \mathrm{me} 1$ relative to H3K4me3 have previously been used to distinguish promoters from enhancers (Natoli and Andrau, 2012). Therefore we looked at histone modifications using chromatin immunoprecipitation (ChIP)-Seq (S2A). We found higher levels of $\mathrm{H} 3 \mathrm{~K} 4 \mathrm{me} 3$ compared to $\mathrm{H} 3 \mathrm{~K} 4 \mathrm{me} 1$ which is indicative of a promoter as opposed to an enhancer. H3K327ac signal was higher then H3K4me3 or H3K4me1 and H3K327me3 was basically absent.

As Gm15551 is expressed antisense from a locus within intron 2 of the intracellular $\mathrm{Ca}^{2+}$ signalling protein Camk2d and it is known that lncRNA can work as in cis regulators of nearby coding genes (Gil and Ulitsky, 2019), we checked whether the expression of Gm15551 correlates with the expression of Camk2d in various publicly available RNA-Seq datasets of adipose tissue, but found no significant correlation (ANCOVA, $p=0.848$; Fig S2B). To exclude the possibility of Gm15551 being a coding gene wrongly annotated as lncRNA (Anderson et al.,2015), we calculated the coding potential for all genes expressed in our dataset using CPAT (Wang et al.,2013). Gm15551 showed a low coding probability comparable to other known lncRNA genes as opposed to the brown adipocyte marker genes Cidea, Adcy3 and Ucp1 (Fig 2D). Similarly, ranking genes by the ratio of ribosome associated over total RNA in a publicly available TRAP-Seq data set of iBAT sorted Gm15551 with other lncRNA genes (Fig S2C).

Next we followed the gene expression of Gm15551 during the differentiation of preadipocytes into mature adipocytes. Our analysis showed that Gm15551 is highly upregulated already early in differentiation, similar to the core adipocyte transcription factor Pparg and unlike Ucp 1 which only reaches maximum levels in late differentiation (Fig $2 \mathrm{E}$ ). In order to mimic the effects of cold treatment on adipose tissue in vitro, we stimulated cells with the non-selective $\beta$-adrenergic agonist isoproterenol or $\beta_{3}$ specific agonist CL316243. Both stimuli were sufficient to repress Gm15551 in differentiated adipocytes originating from eWAT, iWAT and iBAT (Fig2 2F). In primary immortalized brown adipocytes, the effect of $\beta$-adrenergic stimulation on the expression of Gm15551 was stable over 24h (Fig S2D). 


\section{Gain- and loss-of-function of Gm15551 does not disturb brown adipocyte development and function in vitro}

To investigate the role of Gm15551 in differentiation and function of brown adipocytes, we used an immortalized brown preadipocyte cell line stably expressing the CRISPRa SAM system for gainof-function studies together with locked nucleic acid (LNA) antisense oligonucleotides for loss-offunction studies (Lundh et al., 2017). Transfection of either one of two plasmids encoding single guide RNAs (sgRNAs) targeting Gm15551 two days prior to the induction of the differentiation led to a robust overexpression of Gm15551 compared to the empty vector control at day 1 of differentiation (Fig $3 \mathrm{~A}$ ). The effect of the overexpression was greatly diminished on day 4 and 7 because the natural gene expression of Gm15551 rises during differentiation. However, we could not observe any changes in the expression of common and brown adipocyte marker genes or in the cells ability to accumulate lipids (Fig]A, B, Fig S3A).

Next we set out to knock down Gm15551 in mature adipocytes. In order to detect potential interactions of Gm15551 expression with thermogenic activation of brown adipocytes, we looked at cells both under basal conditions and under $\beta$-adrenergic stimulation. Reverse transfection with two different LNAs targeting Gm15551 on day 4 of differentiation resulted in robust downregulation of Gm15551 on day 7 compared to the non-targeting control LNA (Fig S3B). Overall, we found 2762 genes differentially regulated by either knockdown or stimulation (Fig $3 \mathrm{C}$ ). Hierarchical clustering showed, that the influence of the $\beta$-adrenergic stimulation was more pronounced then that of the loss-of-function of Gm155551. Samples treated with the control non-targeting LNA clustered together with LNA1 treated samples in both the basal and stimulated condition, indicating that the two LNAs used caused different effects. Looking at the specific effect of the each LNA individually, we found 70 and 188 differentially regulated genes respectively (Fig $3 \mathrm{E}$, Fig $33 \mathrm{C}$ ). There was only an overlap of 16 genes detected to be significantly regulated by the knockdown of Gm15551 using either of the two LNAs. Gene ontology (GO) analysis revealed that these genes were enriched for signalling and especially NF-kB mediated signalling (data not shown).

Similarly, reverse transfection with sgRNA encoding plasmids led to a small but significant overexpression of Gm15551 in fully matured adipocytes and noteworthy was able to suppress its downregulation upon $\beta$-adrenergic stimulation (Fig $\mathrm{S3} \mathrm{D}$ ). However, we did not observe any changes in the gene expression of any of the probed adipocyte marker genes. We further raised the overexpression efficiency by simultaneous transfection of two different plasmids encoding sgRNAs targeting Gm15551 (Fig S3E). We sequenced the transcriptomes of these samples and found a total of 792 genes differentially regulated by either gain-of-function of Gm15551 or $\beta$-adrenergic stimulation (Fig 3D). The effect of the thermogenic activation dominated the dataset as shown by hierarchical clustering. However, there were also two clusters with genes affected by the overexpression of Gm15551. When we specifically looked for changes in gene expression caused by the Gm155551 gain-of-function, we found 14 differentially expressed genes and GO analysis showed an enrichment for genes involved in inflammatory response (Fig $3 \mathrm{E}, \mathrm{F})$.

Comparison between the genes differentially regulated by the gain and loss-of-function of Gm15552 showed that there was no overlap. Additionally, most genes affected by the knock down of Gm15551 showed no change in gene expression in the gain-of-function experiment (Fig $\mathrm{S3} \mathrm{H}$ ). Finally, Oil red O staining of mature adipocytes showed no effect of either gain or loss-of-function of Gm15551 on the cells' ability for lipid accumulation (Fig $\mathrm{S3} F$ ). 


\section{Gm15551 loss-of-function does not impair adipose tissue function in vivo}

Next we created a loss-of-function mouse model by knocking out exon 1 of Gm15551. Since brown adipose tissue plays a role in the regulation of body weight as well as lipid and glucose metabolism (Rui, 2017), we challenged homozygous $\Delta \mathrm{Gm} 15551$ mice and wild type litter mates from 8 weeks of age for 12 weeks with a high fat diet and repeatedly measured body weight and performed intraperitoneal glucose tolerance test (IPGTT), and indirect calorimetry (Fig S4A). While the HFD was sufficient to provoke a significant raise in body weight (Fig $4 \mathrm{~A} ; p=0.038)$ characterised by an increased amount of body fat (Fig S4 B; $p=0.027$ ), we did not observe significant changes induced by the lossof-function of Gm15551 ( $p=0.29$ and 0.76 respectively). Similarly, prolonged HFD treatment but not Gm15551 loss-of-function resulted in impaired glucose tolerance (Fig $4 \mathrm{~B}$; no $\mathrm{p}$ as too little n). Additionally, adipocyte diameter and morphology of HFD treated animals was not affected by Gm15551 knockout (Fig S4C, D; $p=0.359$ ). Next we performed indirect calorimetry while sequentially changing the temperature first from room temperature to thermoneutrality $\left(30^{\circ} \mathrm{C}\right)$, followed by a period at $4{ }^{\circ} \mathrm{C}$ before returning to room temperature $\left(23^{\circ} \mathrm{C}\right)$. Upon the beginning of thermoneutrality, energy expenditure slightly dropped and consequently raised when the temperature was dropped. Upon return to room temperature, the energy expenditure went back to the starting point (Fig $4 \mathrm{C}$ ). However, there was no effect of the Gm15551 knock out (no statistics done so far because to little number of animals). Respiratory exchange ratio of control diet animals raised with the onset of the first dark phase at thermoneutrality and dropped again in the following light phase indicating combustion of carbohydrates taken up with the food during dark phase(Fig 4D). With prolonged cold treatment, the respiratory exchange rate rose again to an intermediate value indicating the mice had to take up food in addition to combusting stored lipids. At the end of the cold treatment, the respiratory exchange rate rose even further indicative of the mice mostly relying on energy from the taken up carbohydrates. This effect of temperature and day light cycle was mostly suppressed in HFD animals as they take up less carbohydrates with their alimentation. Again there was no evidence of an impact of the Gm15551 loss-of-function (no statistics done).

When we compared the adipose tissue transcriptomes from HFD and control diet fed animals, we found 5655 differentially expressed genes (LRT, $p<0.001$; Fig 4 C). Hierarchical clustering was strongly driven by the difference between brown and white adipose tissues. Gene ontology analysis showed that the genes with higher expression in brown adipose tissue were enriched for the terms related to mitochondria, while genes showing a higher expression in white adipose tissue were enriched for terms related to immune system and locomotion (Fig $\mathrm{S} 4 \mathrm{E}$ ). The direct comparison of the samples from wild type animals with those from knock out animals revealed only 11 differentially regulated genes (wald test, $s<0.05 ; \widehat{S} 4 \mathrm{~F}$ ). Additionally, we compared the gene expression for iBAT from wild type and $\Delta \mathrm{Gm} 15551$ mice both at room temperature and after $24 \mathrm{~h}$ of cold treatment. Overall, there were 2531 differentially expressed genes (LRT, $p<0.001$ ), which clustered the samples by temperature but not genotype $4 \mathrm{~F}$ ). Both sets of up and down regulated genes upon cold treatment showed enrichment for terms related to metabolism $[\mathrm{S} 4 \mathrm{G})$. Also the direct comparison of the wild type transcriptomes with those from $\Delta \mathrm{Gm} 15551$ animals only revealed 6 differentially expressed genes (wald test, $s<0.05 ; \mathrm{S} 4 \mathrm{H}$ ). 


\section{Discussion}

Cold and nutrient activated BAT regulates energy homeostasis and improves metabolic status via non-shivering thermogenesis and the secretion of endocrine factors (Betz and Enerbäck, 2017; Scheele and Wolfrum, 2020). lncRNAs have been shown to be tissue specific fine tuning regulators of tissue function, and therefore have been proposed as potential selective targets for the treatment of different diseases (Matsui and Corey, 2017; Wahlestedt, 2013). In the recent years, the function of some IncRNAs expressed in adipose tissue has been described (reviewed by Sun and Lin, 2019). However, the function of most lncRNAs remains unknown. Here, we detected a set of $65 \operatorname{lncRNAs}$ whose expression is specific to adipose tissue and correlates with BAT function and characterised Gm15551 further in vitro and in vivo.

We found Gm15551 to be highly adipose tissue specific with a higher expression in white compared to brown adipose tissues. Gm15551 is highly induced in the early stages of brown adipogenesis and downregulated upon beta adrenergic stimulation in both white and brown adipocytes. We could show that the key transcription factor Prdm16, which controls the determination of brown adipocytes and the browning of white adipose tissue (Seale et al., 2011, 2007), binds to the Gm15551 locus in iBAT. Further, we found the Gm15551 locus to be occupied by Pparg in white as well as brown adipose tissues with a more pronounced occupancy in white compared to brown adipose tissue. Pparg is a transcription factor involved in the maintenance of the general adipocyte phenotype but also showing depot specific binding patterns (Siersbæk et al., 2012). These findings led us to hypothesize an adipocyte specific function of Gm15551.

Previous studies have shown that lncRNAs might give rise to unidentified translation products (Anderson et al., 2015; Ji et al., 2015). We used a sequence based bioinformatics tool to calculate coding probability and analysed a public TRAP-Seq data set to detect ribosome associated RNAs. Our results showed that Gm15551 has a low coding probability and is not associated with ribosomes. While enhancers are known to give rise to bidirectionally transcribed, short, unspliced and unstable enhancer RNAs (eRNAs), it has recently been reported that some enhancers can also be the place of unidirectional transcription giving rise to spliced lncRNAs (Gil and Ulitsky, 2018; Natoli and Andrau, 2012). The Gm15551 locus featured a low ratio of the H3K4me1 over the H3K4me3 histone mark, indicative of promoters. However, the Gm15551 locus also features H3K27ac histone marks, high levels of which are characteristic for enhancers (Natoli and Andrau, 2012). Enhancers are cis-regulatory elements, regulating the expression of nearby genes. Therefore we compared the expression of Gm15551 and Camk2d, which overlaps with Gm15551 in the genome, in several adipocyte related RNA-Seq datasets but found no correlation. However, we cannot rule out a potential enhancer function of Gm15551.

In order to unveil potential effects of Gm15551 gain-of-function on brown adipogenesis, we overexpressed Gm15551 two days prior to the induction of differentiation in a brown preadipocyte cell line, but could not measure any effect of the overexpression neither on common and brown adipocyte marker genes nor on lipid accumulation, both under basal conditions and under $\beta$-adrenergic stimulation. Likewise, there was no effect on lipid accumulation in the subsequent gain- and lossof-function experiments in mature brown adipocytes. On transcriptome level, we hypothesised that gain- and loss- of function of Gm15551 should lead to opposite effects on the gene expression of potential target genes of Gm15551. However, there were no genes that were significantly regulated in both datasets. Furthermore, most genes differentially regulated in one dataset did not even show a 
(non-significant) regulation in the other one. Those genes that were oppositely regulated by Gm15551 gain- and loss-of-function such as Lcn2, Saa3, and Hp are inflammatory markers (Maffei et al., 2016; Sommer et al., 2009, 2008). We have previously found them to be differentially regulated by other sgRNAs in several datasets using the wt1-SAM model system and therefore interpret them as a model specific artefact.

As impaired iBAT function has been shown to render mice susceptible to diet-induced obesity and insulin intolerance (Guerra et al., 2001; Lowell et al., 1993), we put $\triangle$ Gm15551 mice on HFD for 12 weeks and additionally repeatedly tested their response to cold treatment by indirect calorimetry. While both the prolonged HFD treatment and sex caused clear differences, we could not detect any significant effect of the Gm15551 loss-of-function on the examined adipose tissue and metabolic parameters such as energy expenditure, body weight and glucose tolerance. However, as the dataset is currently not well balanced, several of the experiments could so far not be statistically analysed. The last two cohorts are expected to be analysed in early 2022. When we analysed adipose tissue transcriptomes, clear differences between the white and brown adipose tissues as well as between iBAT from cold treated and control animals became evident. However, the Gm15551 knockout only caused a minor number of differentially expressed genes, not exceeding what is expected as false positives.

We have identified a set of adipose tissue specific, HFD and cold regulated lncRNAs from which we characterised Gm15551. Albeit it is highly upregulated during brown adipogenesis and its expression correlates with iBAT activity, we could not detect a phenotype of either gain or loss-of-function of Gm15551 in vitro. Likewise we could not detect a detect a Gm15551 related phenotype when performing comprehensive transcriptomic and adipose tissue physiologic phenotyping in vivo. In conclusion, our findings indicate that Gm15551 is dispensable for iBAT development and function, despite its marked upregulation during initial adipose tissue development. This result is in concordance with a study, which previously found Gm15551 to be upregulated in both white and brown adipogenesis, but detected no effect of small interfering RNA (siRNA) mediated knockdown of Gm15551 on white adipocyte differentiation (Sun et al., 2013). While we have not ruled out a potential effect of a knockdown of Gm15551 in brown preadipocytes on adipogenesis, the lack of a phenotype in white adipogenesis as well as in vivo investigations makes it appear implausible to find a phenotype in brown adipogenesis. Functional redundancy has been reported for coding genes such as CD34 and for duplicated genes in general (Hughes et al., 2020: Qian et al., 2010). Furthermore, IncRNAs have been shown to have tissue and cell state dependent and potentially very subtle functions (Derrien et al., 2012). We cannot rule out the possibility of the existance of other genes showing functional redundancy to Gm15551 hiding any effects of the Gm15551 loss-of-function. It was recently reported that in mouse and zebra fish several lncRNAs, which were selected because of their high expression levels, conservation or because they were located proximal to known coding developmental regulatory genes, had no effect on embryogenesis, viability and fertility (Goudarzi et al., 2019; Han et al., 2018). In conclusion, while Gm15551 is specifically expressed in adipose tissues and we subjected mice both to HFD and cold treatment, two major stressors of adipose tissue (Alcalá et al., 2017; Sanchez-Gurmaches et al., 2016), it is possible that Gm15551 exhibits either a very subtle function undetectable by our measurements, a context-dependent function in a specific cellular state that we have not investigated, or it might also be that Gm15551 has no biological function in murine adipose tissue. 


\section{Material and methods}

\section{Animal experiments}

Unless otherwise stated, mice were kept at $22^{\circ} \mathrm{C}$ to $24^{\circ} \mathrm{C}$ on a regular $12 \mathrm{~h}$ light cycle with ad libitum access to food and water. Wild type C57BL/6N mice used for the detection of differentially regulated genes and ChIP-Seq in iBAT were fed chow diet (Ssniff V1554) up to the age of 8 weeks, where the respective cohorts were put on HFD (Ssniff D12492 (I) mod.) for 12 weeks and kept at $4{ }^{\circ} \mathrm{C}$ for $24 \mathrm{~h}$. $\Delta \mathrm{Gm} 15551$ mice used for transcriptomics analyses were fed chow diet (Altromin 1324). $\Delta \mathrm{Gm} 15551$ mice used for metabolic phenotyping were fed chow diet (Altromin 1314). Respective cohorts were put on high fat diet (Ssniff D12492 (I) mod.) for 12 weeks starting from 8 weeks of age.

\section{Generation of Gm15551 knock out animals}

The mouse model for genetic deficiency of Gm15551 was generated at the Czech Centre for Phenogenomics using CRISPR/Cas9 to achieve a $1113 \mathrm{bp}$ deletion including exon 1 of Gm15551 on the background of C57BL/6N.

\section{Indirect calorimetry}

Prior to the experiment, a complete calibration protocol for the gas analysers was run according to the manufacturer's recommendations and mice were weighed. The mice were singly housed in a PhenoMaster device (TSE systems) at a regular $12 \mathrm{~h}$ light cycle and $55 \%$ relative humidity with ad libitum access to water and the respective diet. At 11, 15 and 19 weeks of age, mice underwent a temperature challenge starting at $23^{\circ} \mathrm{C}$, followed by $6 \mathrm{~h}$ at $30^{\circ} \mathrm{C}, 18 \mathrm{~h}$ at $4{ }^{\circ} \mathrm{C}$ and $9 \mathrm{~h}$ at $23^{\circ} \mathrm{C}$ again. Sampling rate was $15 \mathrm{~min}$.

\section{IPGTT}

Animals were fasted overnight (16h to $18 \mathrm{~h}$ ) with free access to water. After weighing, the mice received $2 \mathrm{~g} \mathrm{~kg}^{-1}$ i.p. glucose. Blood glucose was measured fasted and after 15, 30, 60 and $120 \mathrm{~min}$ using a standard glucometer.

\section{Adipocyte diameter}

Hematoxylin and eosin staining was performed on tissue slices and slides were scanned. Two representative areas per tissue were exported and analysed using adiposoft (Galarraga et al., 2012).

\section{RNA isolation and reverse transcription}

Cells or frozen tissue samples were homogenised and lysed in TRIsure (Bioline). Total RNA was isolated using EconoSpin All-In-One Mini Spin Columns (EconoSpin 1920-250) and reverse transcribed into cDNA using the High Capacity cDNA Reverse Transcription Kit (Applied Biosystems 4368814) following the manufacturer's instructions. 


\section{Quantitative polymerase chain reaction (qPCR)}

qPCR was performed in 384 well format in a LightCycler $480 \mathrm{II}$ (Roche). $4 \mu \mathrm{l}$ of 1:20 diluted cDNA, $0.5 \mu \mathrm{l}$ gene specific primer mix ( $5 \mu \mathrm{M}$ each) and $4.5 \mu \mathrm{l} \mathrm{FastStart} \mathrm{Essential} \mathrm{cDNA} \mathrm{Green} \mathrm{Master} \mathrm{(Roche)}$ were amplified using 45 cycles of $25 \mathrm{~s}$ at $95^{\circ} \mathrm{C}, 20 \mathrm{~s}$ at $58^{\circ} \mathrm{C}$ and $20 \mathrm{~s}$ at $72^{\circ} \mathrm{C}$ after $300 \mathrm{~s}$ at $95^{\circ} \mathrm{C}$ initial denaturation. All combinations of primers and samples were run in duplicates and $C_{q}$ values calculated as the second derivative maximum. Genes of interest were normalised against housekeeper genes using the $\Delta C_{q}$ method. The primers used in this study can be found in Tab $\mathrm{S2}$

\section{Total RNA sequencing}

RNA sequencing and library preparation were performed at the Cologne Center for Genomics (Cologne, Germany) according to their standard protocols. Before RNA sequencing, rRNA was depleted according to the instructions of the Illumina TruSeq kit. All sequencing experiments were accomplished with a paired-end protocol and a depth resulting in $50 \times 10^{6}$ to $75 \times 10^{6}$ paired reads per sample. Before RNA sequencing, genomic DNA was eliminated following the instructions of the TURBO DNA-freeTM Kit and subsequently $1 \mu l$ RNA was used to examine RNA integrity in an Agilent 2100 Bioanalyzer Analysis System.

\section{Poly A RNA sequencing}

Paired end libraries were constructed using the NEBNext Ultra II RNA Library Prep Kit for Illumina following the manufacturer's protocol and sequenced on an Illumina NovaSeq 6000 in 2 x 50-bp paired end reads.

\section{RNA sequencing data analysis}

Reads were quality filtered using cutadapt (Martin, 2011). For visualisation, reads were mapped to the GRCm38 genome using STAR (Dobin et al.,2013). For quantification, reads were mapped to the Gencode M22 transcriptome or a combination of M22 and RNAcentral 5 using salmon (Patro et al., 2017).

\section{ChIP sequencing}

For histone modification sequencing, brown adipose tissues of two mice were used each sequencing experiment. Prior to ChIP, BAT was dissociated using a gentleMACSTM Dissociator (Miltenyi biotec, Germany). The cell suspension was cross linked with $1 \%$ formaldehyde for 10 min at RT and the reaction was quenched with $0.125 \mathrm{M}$ glycine for $5 \mathrm{~min}$ to $10 \mathrm{~min}$ at RT. Cells were washed twice with cold PBS and PMSF and snap-frozen in liquid nitrogen before storing at $-80^{\circ} \mathrm{C}$.

Frozen pellets were thawed on ice for $30 \mathrm{~min}$ to $60 \mathrm{~min}$. Pellets were resuspended in $5 \mathrm{ml}$ lysis buffer 1 ( $50 \mathrm{~mm}$ Hepes, $140 \mathrm{~mm} \mathrm{NaCl}, 1 \mathrm{~mm}$ EDTA, $10 \%$ glycerol, $0.5 \%$ NP- $40,0.25 \%$ Triton X-100) by pipetting and then rotated vertically at $4{ }^{\circ} \mathrm{C}$ for $10 \mathrm{~min}$. Pellets were resuspended in $5 \mathrm{ml}$ lysis buffer 2 (10 mM Tris, $200 \mathrm{mM} \mathrm{NaCl}, 1 \mathrm{~mm}$ EDTA, $0.5 \mathrm{mM}$ EGTA) and incubated at vertical rotation and at room temperature for $10 \mathrm{~min}$. Samples were centrifuged for $5 \mathrm{~min}$ at $1350 \mathrm{~g}$ at $4{ }^{\circ} \mathrm{C}$ and supernatant was carefully aspirated. Then, samples were resuspended in $3 \mathrm{ml}$ lysis buffer $3(10 \mathrm{mM}$ Tris, $100 \mathrm{mM} \mathrm{NaCl}$, $1 \mathrm{mM}$ EDTA, $0.5 \mathrm{mM}$ EGTA, $0.1 \%$ Na-deoxycholate, $0.5 \%$ N-lauroylsarcosine) and were separated into 2 times $1.5 \mathrm{ml}$ in $15 \mathrm{ml}$ polypropylene tubes, in which they were sonicated with the following 
settings by Bioruptorő Plus sonication: power $=$ high, on interval $=30 \mathrm{~s}$, off interval $=45 \mathrm{~s}$, total time $=10 \mathrm{~min}$ (18 cycles of on/off). Sonicated samples were transferred to a $1.5 \mathrm{ml}$ microfuge tube and were centrifuged for $10 \mathrm{~min}$ at $16000 \mathrm{~g}$ at $4{ }^{\circ} \mathrm{C}$ to pellet cellular debris. $10 \%$ of sample solution were stored to be used as input control, while the rest was used for ChIP.

To capture different histone modifications, $5 \mu \mathrm{g}$ to $10 \mu \mathrm{g}$ of the respective antibodies (Tab S3) were added to the sonicated ChIP reaction and rotated vertically at $4{ }^{\circ} \mathrm{C}$ overnight. $100 \mu$ Dynabeads (Protein A or Protein G) for each ChIP sample were prepared according to the manufacturer's instructions, mixed with $1 \mathrm{ml}$ of antibody-bound chromatin and rotated vertically at $4{ }^{\circ} \mathrm{C}$ for at least $2 \mathrm{~h}$ to $4 \mathrm{~h}$. Bound beads were washed at least five times in $1 \mathrm{ml}$ cold RIPA $(50 \mathrm{mM}$ Hepes, $500 \mathrm{mM} \mathrm{LiCl}$, $1 \mathrm{mM}$ EDTA, $1 \%$ NP-40, 0.7\% Na-deoxycholate) and once in $1 \mathrm{ml}$ cold TE buffer containing $50 \mathrm{mM}$ $\mathrm{NaCl}$. Samples were eluted for $15 \mathrm{~min}$ with elution buffer (50 mM Tris, $10 \mathrm{~mm}$ EDTA, $1 \%$ SDS) at $65^{\circ} \mathrm{C}$ and continuously shaken at $700 \mathrm{~min}^{-1}$. Beads were separated using a magnet and $200 \mu \mathrm{l}$ supernatant were transferred to fresh microfuge tubes. Input samples were thawed and mixed with $300 \mu l$ elution buffer. $\mathrm{ChIP} /$ input samples were incubated at $65^{\circ} \mathrm{C}$ in a water bath overnight to reverse the cross linking reaction. TE buffer was added at room temperature to dilute SDS in both ChIP and input samples. For digestion of RNA and protein contamination, RNase A was added to the samples and incubated in a $37^{\circ} \mathrm{C}$ water bath for $2 \mathrm{~h}$; then proteinase $\mathrm{K}$ was added to a final concentration of $0.2 \mathrm{mg} \mathrm{ml}^{-1}$ and incubated in a $55^{\circ} \mathrm{C}$ water bath for $2 \mathrm{~h}$. Finally, DNA was extracted using a standard phenol-chloroform extraction method at room temperature and DNA concentrations were measured using a NanoDrop ND-1000 spectrophotometer or Qubit dsDNA HS Assay Kit and stored at $-80^{\circ} \mathrm{C}$ until sequencing.

\section{ChIP sequencing data analysis}

Reads were mapped to the GRCm38 genome using bowtie2 (Langmead and Salzberg, 2012) after quality filtering by cutadapt.

\section{Tissue specificity}

Tissue specificity scores were calculated for every gene over seven metabolically active tissues as $\frac{\log _{2}\left(T P M_{n}+1\right)}{\sum \log _{2}\left(T P M_{n}+1\right)}$ as described by (Alvarez-Dominguez et al., 2015) from RNA-Seq data that we have previously published (GEO: GSE121345

\section{Gene set enrichment analysis}

Gene set enrichment was done using topGO for GO (Alexa et al., 2006) and ReactomePA for reactome (Yu and $\mathrm{He}, 2016$ ).

\section{Assessment of coding potential}

Coding potential was calculated using CPAT (Wang et al.,2013). Ribosome scores were calculated as $\log 2$ (TRAP/totalRNA), where TRAP are the normalised counts from a publicly available dataset of translating ribosomal affinity purification (TRAP) of mouse iBAT (GEO: GSE103617) and totalRNA are the normalised counts from the room temperature control diet iBAT total RNA samples. 


\section{Primary cell culture}

Inguinal and epididymal white as well as intrascapular brown adipose tissues from 6 to 8 week old C57BL/6J mice were dissected, minced and digested with collagenase II (worthington) and dispase II (Sigma, iBAT only). Cells were seeded in 24 well plates and grown in DMEM/Ham's F12 medium supplemented with $0.1 \%$ Biotin/D-Pantothenate $(33 \mathrm{~mm} / 17 \mathrm{mM}), 1 \%$ penicillin-streptomycin and $20 \%$ FBS. Upon reaching confluency, FBS concentration was reduced to $10 \%$ and differentiation was induced using $1 \mu \mathrm{M}$ rosiglitazone, $850 \mathrm{nM}$ insulin, $1 \mu \mathrm{M}$ dexamethasone, $250 \mu \mathrm{M}$ 3-isobutyl-1-methylxanthin (IBMX), $125 \mu \mathrm{M}$ indomethacine (brown only) and $1 \mathrm{nM}$ triiodothyronine (T3) (iBAT only). Subsequently, medium was changed every other day for medium containing $10 \%$ FBS, rosiglitazone and T3 (iBAT only). Full differentiation was reached 7 days after induction. Cells were stimulated using $1 \mu \mathrm{M}$ isoproterenol or $10 \mu \mathrm{M}$ CL316243.

\section{Cultivation of brown adipocyte cell lines}

The wt1-SAM brown preadipocyte cell line was a kind gift from Dr. Brice Emanuelli. The cells were grown in high glucose DMEM supplemented with $10 \%$ FBS and $1 \%$ penicillin-streptomycin. After reaching confluency, differentiation was induced by $0.5 \mu \mathrm{M}$ rosiglitazone, $1 \mathrm{nM} \mathrm{T} 3,1 \mu \mathrm{M}$ Dexamethasone, $850 \mathrm{nM}$ insulin, $125 \mu \mathrm{M}$ indomethacine and $500 \mu \mathrm{M}$ IBMX. Two days later, medium was exchanged for medium supplemented with $0.5 \mu \mathrm{M}$ rosiglitazone and $850 \mathrm{nM}$ insulin. Afterwards, medium was changed for medium containing $0.5 \mu \mathrm{M}$ rosiglitazone every second day. Full differentiation was reached 7 days after induction.

PIBA cells were cultured in the same medium as wt1-SAM cells. A common induction/stimulation cocktail consisting of $10 \mu \mathrm{M}$ rosiglitazone, $1 \mathrm{nM}$ T3, $0.5 \mu \mathrm{M}$ Dexamethasone, $850 \mathrm{nM}$ insulin, $12.5 \mu \mathrm{M}$ indomethacine and $125 \mu \mathrm{M}$ IBMX was used to differentiate cells.

\section{In vitro gain and loss of function studies}

For in vitro gain of function studies using the wt1-SAM cell line, sgRNAs were designed using CRISPick (Doench et al., 2014) and cloned into the sgRNA(MS2) cloning backbone (addgene 61424) as described by Konermann et al. (2015). Empty vector and Ucp1 targeting sgRNAs were used as controls and were kind gifts of Dr. Brice Emanuelli (Lundh et al.,2017). Target sequences used are found in TabS1.

LNA gapmers designed and synthesized by Qiagen were used for in vitro loss of function experiments. Two non targeting scrambled LNAs were used as control.

Preadipocytes were transfected by seeding 30000 cells per well of a 24 well plate in growth medium and adding $1.5 \mu \mathrm{l}$ TransIT-X2 (Mirus) and $125 \mathrm{ng}$ plasmid DNA or $1.4 \mu \mathrm{l}$ LNA $(10 \mu \mathrm{M})$ in $50 \mu \mathrm{l}$ OptiMEM I once the cells had attached.

In order to transfect mature adipocytes, $3 \mu \mathrm{l}$ TransIT and $250 \mathrm{ng}$ plasmid DNA or $1.4 \mu \mathrm{l}(10 \mu \mathrm{M})$ in $100 \mu \mathrm{l}$ Opti-MEM I were pipetted into a well of a 24 well plate. After $15 \mathrm{~min}, 500000$ cells resuspended in $500 \mu \mathrm{l}$ Opti-MEM were added. $24 \mathrm{~h}$ later, medium was changed for regular differentiation medium.

\section{Oil red $O$ staining}

Cells were fixed with $4 \%$ formalin for $30 \mathrm{~min}$, rinsed once with water followed by $60 \%$ isopropanol. Cells were stained with Oil Red O (0.3\% in $60 \%$ isopropanol) for $10 \mathrm{~min}$. Excess dye was rinsed with 
water. For quantification, the Oil Red O was eluted in $100 \%$ isopropanol and OD measured at $520 \mathrm{~nm}$ in a multi plate reader.

\section{Statistical analysis}

Statistics for RNA-Seq data was done in DESeq2 (Love et al., 2014) using LRTs for factors with multiple levels or for analysing multiple factors at once and wald tests otherwise. Log fold changes were shrunken and s-values calculated using apeglm (Zhu et al., 2018). Data from animal experiments with repeated measurements were averaged over temperature and day/night conditions and analysed using mixed-effects models with the body weight as cofactor and individual animal as random variable. Other data was analysed using Student's $t$-tests and adjusted for multiple testing using Holm's method. Shown are individual values in addition to mean \pm standard error of the mean.

\section{Acknowledgements}

CH was funded by the National Taiwan University and the grant 109-2917-I-002-029 from the Ministry of Science and Technology, Taiwan. The authors used services of the Czech Centre for Phenogenomics supported by the Czech Academy of Sciences RVO 68378050 and by the project LM2018126 Czech Centre for Phenogenomics provided by Ministry of Education, Youth and Sports of the Czech Republic. JWK and CHE were funded by the University of Southern Denmark and the Danish Diabetes Academy, which is in turn funded by the Novo Nordisk Foundation.

\section{Author contributions}

CHE performed experiments with primary adipocytes, Gm15551 gain of function experiments in preadipocytes, bioinformatic analyses, analysed the data, designed the study and wrote the manuscript. $\mathrm{CH}$ performed the Gm15551 gain and loss of function experiments in mature adipocytes and helped with the $\Delta \mathrm{Gm} 15551$ loss of function experiments for transcriptomics. SK performed the experiments with wild type mice used for transcriptomics and performed ChiP. PK generated the $\Delta$ Gm15551 knockout mouse model. JP, JR and DPR planned and performed the metabolic phenotyping of the $\Delta \mathrm{Gm} 15551$ mice. RS supervised the generation and metabolic phenotyping of the $\Delta \mathrm{Gm} 15551$ mice. JWK designed and supervised the study and wrote the manuscript.

\section{Conflict of interest}

The authors declare no competing interest.

\section{References}

Alcalá, M., Calderon-Dominguez, M., Bustos, E., Ramos, P., Casals, N., Serra, D., Viana, M. and Herrero, L. (22nd Nov. 2017). Increased inflammation, oxidative stress and mitochondrial respiration in brown adipose tissue from obese mice. Scientific Reports 7(1), 16082. DOI: 10 . 1038/s41598017-16463-6. 
Alexa, A., Rahnenführer, J. and Lengauer, T. (1st July 2006). Improved scoring of functional groups from gene expression data by decorrelating GO graph structure. Bioinformatics 22(13), 1600-1607. DOI: $10.1093 /$ bioinformatics/btl140

Alvarez-Dominguez, J.R., Bai, Z., Xu, D., Yuan, B., Lo, K.A., Yoon, M.J., Lim, Y.C., Knoll, M., Slavov, N., Chen, S., Peng, C., Lodish, H.F. and Sun, L. (5th May 2015). De novo Reconstruction of Adipose Tissue Transcriptomes Reveals Novel Long Non-coding RNAs that Regulate Brown Adipocyte Development. Cell Metab 21(5), 764-776. DOI: 10.1016/j.cmet.2015.04.003.

Anderson, D.M., Anderson, K.M., Chang, C.-L., Makarewich, C.A., Nelson, B.R., McAnally, J.R., Kasaragod, P., Shelton, J.M., Liou, J., Bassel-Duby, R. and Olson, E.N. (12th Feb. 2015). A Micropeptide Encoded by a Putative Long Noncoding RNA Regulates Muscle Performance. Cell 160(4), 595-606. DOI: $10.1016 / \mathrm{j}$.cell.2015.01.009.

Angelantonio, E.D., Bhupathiraju, S.N., Wormser, D., Gao, P., Kaptoge, S., Gonzalez, A.B.d., Cairns, B.J., Huxley, R., Jackson, C.L., Joshy, G., Lewington, S., Manson, J.E., Murphy, N., Patel, A.V., Samet, J.M., Woodward, M., Zheng, W., Zhou, M., Bansal, N., Barricarte, A. et al. (20th Aug. 2016). Bodymass index and all-cause mortality: individual-participant-data meta-analysis of 239 prospective studies in four continents. The Lancet 388(10046). Publisher: Elsevier, 776-786. DOI: 10 . $1016 /$ S0140-6736(16)30175-1

Bai, Z., Chai, X.-r., Yoon, M.J., Kim, H.-J., Lo, K.A., Zhang, Z.-c., Xu, D., Siang, D.T.C., Walet, A.C.E., Xu, S.-h., Chia, S.-Y., Chen, P., Yang, H., Ghosh, S. and Sun, L. (1st Aug. 2017). Dynamic transcriptome changes during adipose tissue energy expenditure reveal critical roles for long noncoding RNA regulators. PLOS Biology 15(8). tex.ids: bai_dynamic_2017, e2002176. DOI: 10.1371/ journal.pbio.2002176

Bast-Habersbrunner, A., Kiefer, C., Weber, P., Fromme, T., SchieSSl, A., Schwalie, P.C., Deplancke, B., Li, Y. and Klingenspor, M. (31st May 2021). LncRNA Ctcflos orchestrates transcription and alternative splicing in thermogenic adipogenesis. EMBO reports $n / a(n / a)$. Publisher: John Wiley \& Sons, Ltd, e51289. DOI: 10.15252/embr . 202051289

Betz, M.J. and Enerbäck, S. (23rd Oct. 2017). Targeting thermogenesis in brown fat and muscle to treat obesity and metabolic disease. Nature Reviews Endocrinology, nrendo.2017.132. DOI: 10 . $1038 /$ nrendo.2017.132

Cannon, B. and Nedergaard, J. (1st Jan. 2004). Brown Adipose Tissue: Function and Physiological Significance. Physiological Reviews 84(1), 277-359. DOI: 10.1152/physrev.00015.2003

Derrien, T., Johnson, R., Bussotti, G., Tanzer, A., Djebali, S., Tilgner, H., Guernec, G., Martin, D., Merkel, A., Knowles, D.G., Lagarde, J., Veeravalli, L., Ruan, X., Ruan, Y., Lassmann, T., Carninci, P., Brown, J.B., Lipovich, L., Gonzalez, J.M., Thomas, M. et al. (9th Jan. 2012). The GENCODE v7 catalog of human long noncoding RNAs: Analysis of their gene structure, evolution, and expression. Genome Res. 22(9), 1775-1789. DOI: 10.1101/gr.132159.111.

Djebali, S., Davis, C.A., Merkel, A., Dobin, A., Lassmann, T., Mortazavi, A., Tanzer, A., Lagarde, J., Lin, W., Schlesinger, F., Xue, C., Marinov, G.K., Khatun, J., Williams, B.A., Zaleski, C., Rozowsky, J., Röder, M., Kokocinski, F., Abdelhamid, R.F., Alioto, T. et al. (Sept. 2012). Landscape of transcription in human cells. Nature 489(7414). tex.ids: djebali_landscape_2012, 101-108. DOI: $10.1038 /$ nature11233

Dobin, A., Davis, C.A., Schlesinger, F., Drenkow, J., Zaleski, C., Jha, S., Batut, P., Chaisson, M. and Gingeras, T.R. (1st Jan. 2013). STAR: ultrafast universal RNA-seq aligner. Bioinformatics 29(1), 1521. DOI: 10.1093/bioinformatics/bts635. 
Doench, J.G., Hartenian, E., Graham, D.B., Tothova, Z., Hegde, M., Smith, I., Sullender, M., Ebert, B.L., Xavier, R.J. and Root, D.E. (Dec. 2014). Rational design of highly active sgRNAs for CRISPRCas9-mediated gene inactivation. Nat Biotechnol 32(12), 1262-1267. DOI: 10.1038/nbt. 3026

Galarraga, M., Campión, J., Muñoz-Barrutia, A., Boqué, N., Moreno, H., Martínez, J.A., Milagro, F. and Ortiz-de-Solórzano, C. (Dec. 2012). Adiposoft: automated software for the analysis of white adipose tissue cellularity in histological sections. J Lipid Res 53(12), 2791-2796. DOI: $10.1194 / j 1 r$. D023788.

Gil, N. and Ulitsky, I. (28th Nov. 2018). Production of Spliced Long Noncoding RNAs Specifies Regions with Increased Enhancer Activity. Cell Systems 7(5), 537-547.e3. DOI: 10 . 1016 / j . cels . 2018.10 .009

Gil, N. and Ulitsky, I. (15th Nov. 2019). Regulation of gene expression by cis -acting long non-coding RNAs. Nat Rev Genet, 1-16. DOI: 10.1038/s41576-019-0184-5.

Goudarzi, M., Berg, K., Pieper, L.M. and Schier, A.F. (8th Jan. 2019). Individual long non-coding RNAs have no overt functions in zebrafish embryogenesis, viability and fertility. eLife 8. Ed. by E.M. Busch-Nentwich and D.Y. Stainier. Publisher: eLife Sciences Publications, Ltd, e40815. DOI: 10 . 7554/eLife.40815.

Guerra, C., Navarro, P., Valverde, A.M., Arribas, M., Brüning, J., Kozak, L.P., Kahn, C.R. and Benito, M. (15th Oct. 2001). Brown adipose tissuespecific insulin receptor knockout shows diabetic phenotype without insulin resistance. J Clin Invest 108(8). Publisher: American Society for Clinical Investigation, 1205-1213. DOI: $10.1172 /$ JCI13103

Han, X., Luo, S., Peng, G., Lu, J.Y., Cui, G., Liu, L., Yan, P., Yin, Y., Liu, W., Wang, R., Zhang, J., Ai, S., Chang, Z., Na, J., He, A., Jing, N. and Shen, X. (1st Apr. 2018). Mouse knockout models reveal largely dispensable but context-dependent functions of lncRNAs during development. Journal of Molecular Cell Biology 10(2), 175-178. DOI: 10.1093/jmcb/mjy003

Henriques, F., Bedard, A.H., Guilherme, A., Kelly, M., Chi, J., Zhang, P., Lifshitz, L.M., Bellvé, K., Rowland, L.A., Yenilmez, B., Kumar, S., Wang, Y., Luban, J., Weinstein, L.S., Lin, J.D., Cohen, P. and Czech, M.P. (4th Aug. 2020). Single-Cell RNA Profiling Reveals Adipocyte to Macrophage Signaling Sufficient to Enhance Thermogenesis. Cell Rep 32(5), 107998. DOI: 10 .1016/ j celrep . 2020.107998

Hughes, M.R., Canals Hernaez, D., Cait, J., Refaeli, I., Lo, B.C., Roskelley, C.D. and McNagny, K.M. (1st June 2020). A sticky wicket: Defining molecular functions for CD34 in hematopoietic cells. Experimental Hematology 86,1-14. DOI: 10.1016/j . exphem.2020.05.004

Ji, Z., Song, R., Regev, A. and Struhl, K. (19th Dec. 2015). Many lncRNAs, 5UTRs, and pseudogenes are translated and some are likely to express functional proteins. eLife Sciences 4, e08890. DOI: 10.7554/eLife.08890

Klepac, K., Georgiadi, A., Tschöp, M. and Herzig, S. (16th July 2019). The role of brown and beige adipose tissue in glycaemic control. Molecular Aspects of Medicine. DOI: 10.1016/j . mam . 2019 . 07.001 .

Konermann, S., Brigham, M.D., Trevino, A.E., Joung, J., Abudayyeh, O.O., Barcena, C., Hsu, P.D., Habib, N., Gootenberg, J.S., Nishimasu, H., Nureki, O. and Zhang, F. (Jan. 2015). Genome-scale transcriptional activation by an engineered CRISPR-Cas9 complex. Nature 517(7536), 583-588. DOI: 10.1038/nature14136

Langmead, B. and Salzberg, S.L. (Apr. 2012). Fast gapped-read alignment with Bowtie 2. Nature Methods 9(4), 357-359. DOI: 10.1038/nmeth.1923. 
Love, M.I., Huber, W. and Anders, S. (5th Dec. 2014). Moderated estimation of fold change and dispersion for RNA-seq data with DESeq2. Genome Biology 15, 550. DOI: 10 . 1186/s13059-0140550-8

Lowell, B.B., S-Susulic, V., Hamann, A., Lawitts, J.A., Himms-Hagen, J., Boyer, B.B., Kozak, L.P. and Flier, J.S. (Dec. 1993). Development of obesity in transgenic mice after genetic ablation of brown adipose tissue. Nature 366(6457). Bandiera_abtest: a Cg_type: Nature Research Journals Number: 6457 Primary_atype: Research Publisher: Nature Publishing Group, 740-742. DOI: 10 . $1038 /$ 366740a0,

Lundh, M., Pluciñska, K., Isidor, M.S., Petersen, P.S.S. and Emanuelli, B. (1st Oct. 2017). Bidirectional manipulation of gene expression in adipocytes using CRISPRa and siRNA. Molecular Metabolism 6(10), 1313-1320. DOI: 10.1016/j.molmet.2017.07.001

Maffei, M., Barone, I., Scabia, G. and Santini, F. (Aug. 2016). The Multifaceted Haptoglobin in the Context of Adipose Tissue and Metabolism. Endocr Rev 37(4), 403-416. DOI: 10 .1210/er . 20161009

Martin, M. (2nd May 2011). Cutadapt removes adapter sequences from high-throughput sequencing reads. EMBnet.journal 17(1). Number: 1, 10-12. DOI:10.14806/ej.17.1.200.

Matsui, M. and Corey, D.R. (Mar. 2017). Perspectives: Noncoding RNAs as drug targets. Nat Rev Drug Discov 16(3), 167-179. DOI: 10.1038/nrd.2016.117.

Natoli, G. and Andrau, J.-C. (2012). Noncoding Transcription at Enhancers: General Principles and Functional Models. Annual Review of Genetics 46(1), 1-19. DOI: 10.1146/annurev-genet-110711155459

NCD Risk Factor Collaboration (NCD-RisC) (2nd Apr. 2016). Trends in adult body-mass index in 200 countries from 1975 to 2014: a pooled analysis of 1698 population-based measurement studies with 19 ů2 million participants. Lancet 387(10026), 1377-1396. DOI: 10.1016/S0140-6736 (16) 30054-X.

Nedergaard, J., Bengtsson, T. and Cannon, B. (1st Aug. 2007). Unexpected evidence for active brown adipose tissue in adult humans. American Journal of Physiology - Endocrinology and Metabolism 293(2), E444-E452. DOI:10.1152/ajpendo.00691.2006.

Nguyen, T.C., Zaleta-Rivera, K., Huang, X., Dai, X. and Zhong, S. (Nov. 2018). RNA, Action through Interactions. Trends Genet. 34(11), 867-882. DOI: 10.1016/j.tig.2018.08.001

Patro, R., Duggal, G., Love, M.I., Irizarry, R.A. and Kingsford, C. (Apr. 2017). Salmon provides fast and bias-aware quantification of transcript expression. Nature Methods 14(4), 417-419. DOI: 10 . 1038/nmeth. 4197.

Pradas-Juni, M., Hansmeier, N.R., Link, J.C., Schmidt, E., Larsen, B.D., Klemm, P., Meola, N., Topel, H., Loureiro, R., Dhaouadi, I., Kiefer, C.A., Schwarzer, R., Khani, S., Oliverio, M., Awazawa, M., Frommolt, P., Heeren, J., Scheja, L., Heine, M., Dieterich, C. et al. (31st Jan. 2020). A MAFG-lncRNA axis links systemic nutrient abundance to hepatic glucose metabolism. Nat Commun 11(1), 1-17. DOI: $10.1038 / \mathrm{s} 41467-020-14323-\mathrm{y}$.

Prospective Studies Collaboration (28th Mar. 2009). Body-mass index and cause-specific mortality in 900000 adults: collaborative analyses of 57 prospective studies. The Lancet 373(9669), 1083-1096. DOI: $10.1016 / \mathrm{S} 0140-6736(09) 60318-4$

Qian, W., Liao, B.-Y., Chang, A.Y.-F. and Zhang, J. (1st Oct. 2010). Maintenance of duplicate genes and their functional redundancy by reduced expression. Trends in Genetics 26(10), 425-430. DOI: $10.1016 / j \cdot$ tig.2010.07.002 
Rosen, E.D. and Spiegelman, B.M. (16th Jan. 2014). What We Talk About When We Talk About Fat. Cell 156(1), 20-44. DOI: $10.1016 / \mathrm{j}$.cell.2013.12.012

Rui, L. (12th Sept. 2017). Brown and Beige Adipose Tissues in Health and Disease. Compr Physiol 7(4), 1281-1306. DOI: 10.1002/cphy.c170001.

Sanchez-Gurmaches, J., Hung, C.-M. and Guertin, D.A. (May 2016). Emerging Complexities in Adipocyte Origins and Identity. Trends in Cell Biology 26(5), 313-326. DOI: 10.1016/j . tcb. 2016.01.004. Scheele, C. and Wolfrum, C. (1st Feb. 2020). Brown Adipose Crosstalk in Tissue Plasticity and Human Metabolism. Endocrine Reviews 41(1), 53-65. DOI: 10.1210/endrev/bnz007.

Schmidt, E., Dhaouadi, I., Gaziano, I., Oliverio, M., Klemm, P., Awazawa, M., Mitterer, G., FernandezRebollo, E., Pradas-Juni, M., Wagner, W., Hammerschmidt, P., Loureiro, R., Kiefer, C., Hansmeier, N.R., Khani, S., Bergami, M., Heine, M., Ntini, E., Frommolt, P., Zentis, P. et al. (6th Sept. 2018). LincRNA H19 protects from dietary obesity by constraining expression of monoallelic genes in brown fat. Nature Communications 9(1), 3622. DOI: 10.1038/s41467-018-05933-8

Seale, P., Conroe, H.M., Estall, J., Kajimura, S., Frontini, A., Ishibashi, J., Cohen, P., Cinti, S. and Spiegelman, B.M. (4th Jan. 2011). Prdm16 determines the thermogenic program of subcutaneous white adipose tissue in mice. J Clin Invest 121(1). Publisher: American Society for Clinical Investigation, 96-105. DOI: 10.1172/JCI44271.

Seale, P., Kajimura, S., Yang, W., Chin, S., Rohas, L.M., Uldry, M., Tavernier, G., Langin, D. and Spiegelman, B.M. (July 2007). Transcriptional control of brown fat determination by PRDM16. Cell Metab 6(1), 38-54. DOI: $10.1016 / j . c m e t .2007 .06 .001$.

Siersbæk, M.S., Loft, A., Aagaard, M.M., Nielsen, R., Schmidt, S.F., Petrovic, N., Nedergaard, J. and Mandrup, S. (9th Jan. 2012). Genome-Wide Profiling of Peroxisome Proliferator-Activated Receptor in Primary Epididymal, Inguinal, and Brown Adipocytes Reveals Depot-Selective Binding Correlated with Gene Expression. Mol. Cell. Biol. 32(17), 3452-3463. DOI: 10.1128/MCB.00526-12.

Sommer, G., Weise, S., Kralisch, S., Lossner, U., Bluher, M., Stumvoll, M. and Fasshauer, M. (2009). Lipocalin-2 is induced by interleukin-1 in murine adipocytes in vitro. Journal of Cellular Biochemistry 106(1). _eprint: https://onlinelibrary.wiley.com/doi/pdf/10.1002/jcb.21980, 103-108. DOI: $10.1002 / \mathrm{jcb} .21980$.

Sommer, G., Weise, S., Kralisch, S., Scherer, P.E., Lössner, U., Blüher, M., Stumvoll, M. and Fasshauer, M. (2008). The adipokine SAA3 is induced by interleukin-1 in mouse adipocytes. Journal of Cellular Biochemistry 104(6). _eprint: https://onlinelibrary.wiley.com/doi/pdf/10.1002/jcb.21782, 22412247. DOI: $10.1002 / \mathrm{jcb} .21782$

Sun, L., Goff, L.A., Trapnell, C., Alexander, R., Lo, K.A., Hacisuleyman, E., Sauvageau, M., TazonVega, B., Kelley, D.R., Hendrickson, D.G., Yuan, B., Kellis, M., Lodish, H.F. and Rinn, J.L. (26th Feb. 2013). Long noncoding RNAs regulate adipogenesis. PNAS 110(9), 3387-3392. DOI: 10.1073/pnas . 1222643110

Sun, L. and Lin, J.D. (1st May 2019). Function and Mechanism of Long Noncoding RNAs in Adipocyte Biology. Diabetes 68(5). Publisher: American Diabetes Association Section: Perspectives in Diabetes, 887-896. DOI: 10.2337/dbi18-0009.

Wahlestedt, C. (June 2013). Targeting long non-coding RNA to therapeutically upregulate gene expression. Nat Rev Drug Discov 12(6). Bandiera_abtest: a Cg_type: Nature Research Journals Number: 6 Primary_atype: Reviews Publisher: Nature Publishing Group Subject_term: Cancer;RNAi;Technology Subject_term_id: cancer;rnai;technology, 433-446. DOI: $10.1038 /$ nrd4018 
Wang, L., Park, H.J., Dasari, S., Wang, S., Kocher, J.-P. and Li, W. (1st Apr. 2013). CPAT: CodingPotential Assessment Tool using an alignment-free logistic regression model. Nucleic Acids Res 41(6), e74-e74. DOI: 10.1093/nar/gkt006

Yao, R.-W., Wang, Y. and Chen, L.-L. (May 2019). Cellular functions of long noncoding RNAs. Nat Cell Biol 21(5). Bandiera_abtest: a Cg_type: Nature Research Journals Number: 5 Primary_atype: Reviews Publisher: Nature Publishing Group Subject_term: Chromatin;Long non-coding RNAs;RNA metabolism Subject_term_id: chromatin;long-non-coding-rnas;rna-metabolism, 542-551. DOI: 10 . 1038/s41556-019-0311-8.

Yi, W., Li, J., Zhu, X., Wang, X., Fan, L., Sun, W., Liao, L., Zhang, J., Li, X., Ye, J., Chen, F., Taipale, J., Chan, K.M., Zhang, L. and Yan, J. (July 2020). CRISPR-assisted detection of RNAprotein interactions in living cells. Nature Methods 17(7). Number: 7 Publisher: Nature Publishing Group, 685688. DOI: $10.1038 / \mathrm{s} 41592-020-0866-0$

Yu, G. and He, Q.-Y. (26th Jan. 2016). ReactomePA: an R/Bioconductor package for reactome pathway analysis and visualization. Mol. BioSyst. 12(2), 477-479. DOI: 10.1039/C5MB00663E.

Zhang, B., Yang, Y., Xiang, L., Zhao, Z. and Ye, R. (2019). Adipose-derived exosomes: A novel adipokine in obesity-associated diabetes. Journal of Cellular Physiology 234(10). _eprint: https://onlinelibrary.wiley.com/doi/pdf/10.1002/jcp.28354, 16692-16702. DOI:10.1002/jcp. 28354.

Zhu, A., Ibrahim, J.G. and Love, M.I. (2018). Heavy-tailed prior distributions for sequence count data: removing the noise and preserving large differences. Bioinformatics. DOI: 10.1093/bioinformaticis/ bty895 


\section{Figures}

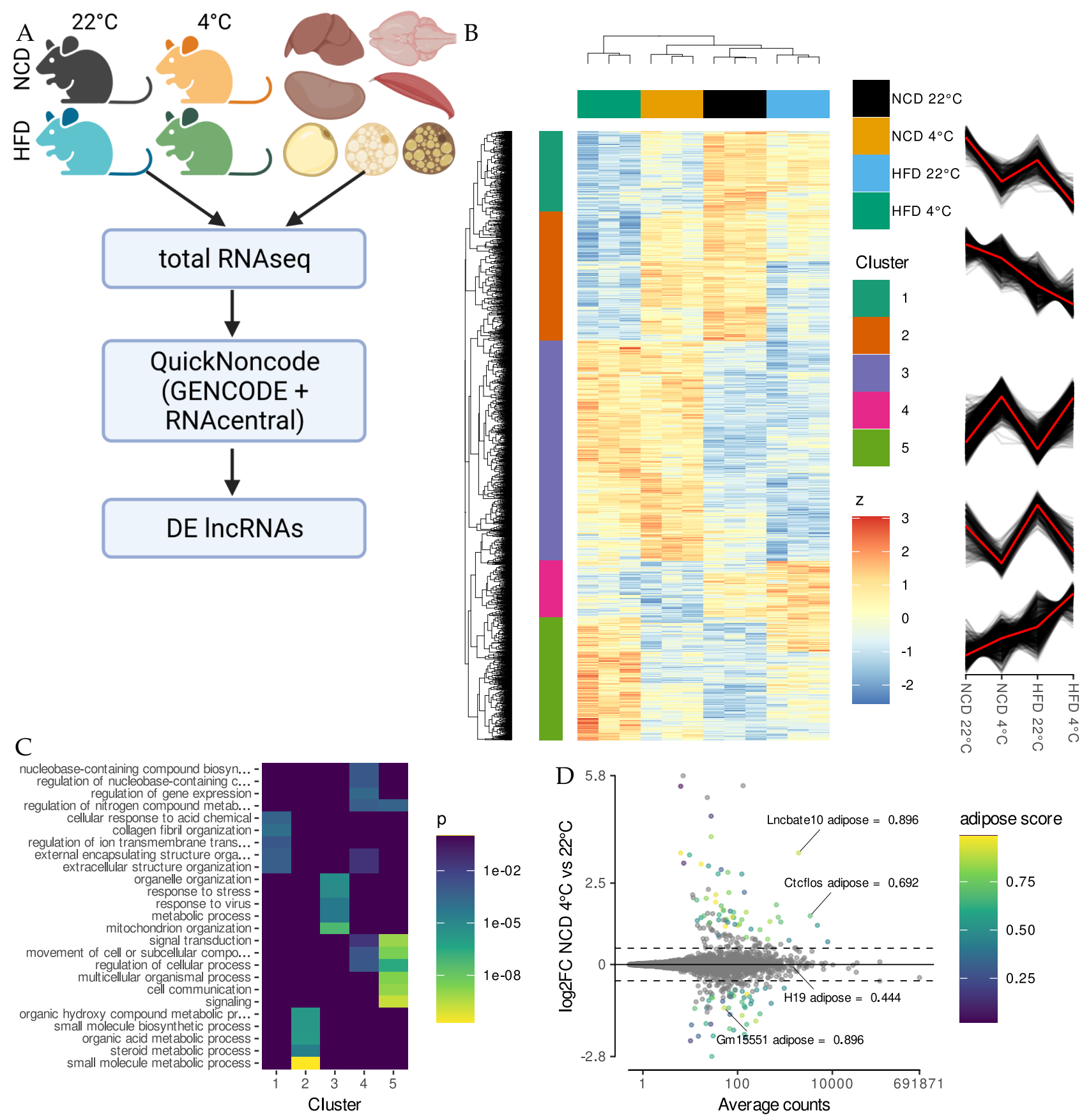

Figure 1: RNA-Seq reveals temperature and obesity dependent changes in iBAT IncRNA expression. 

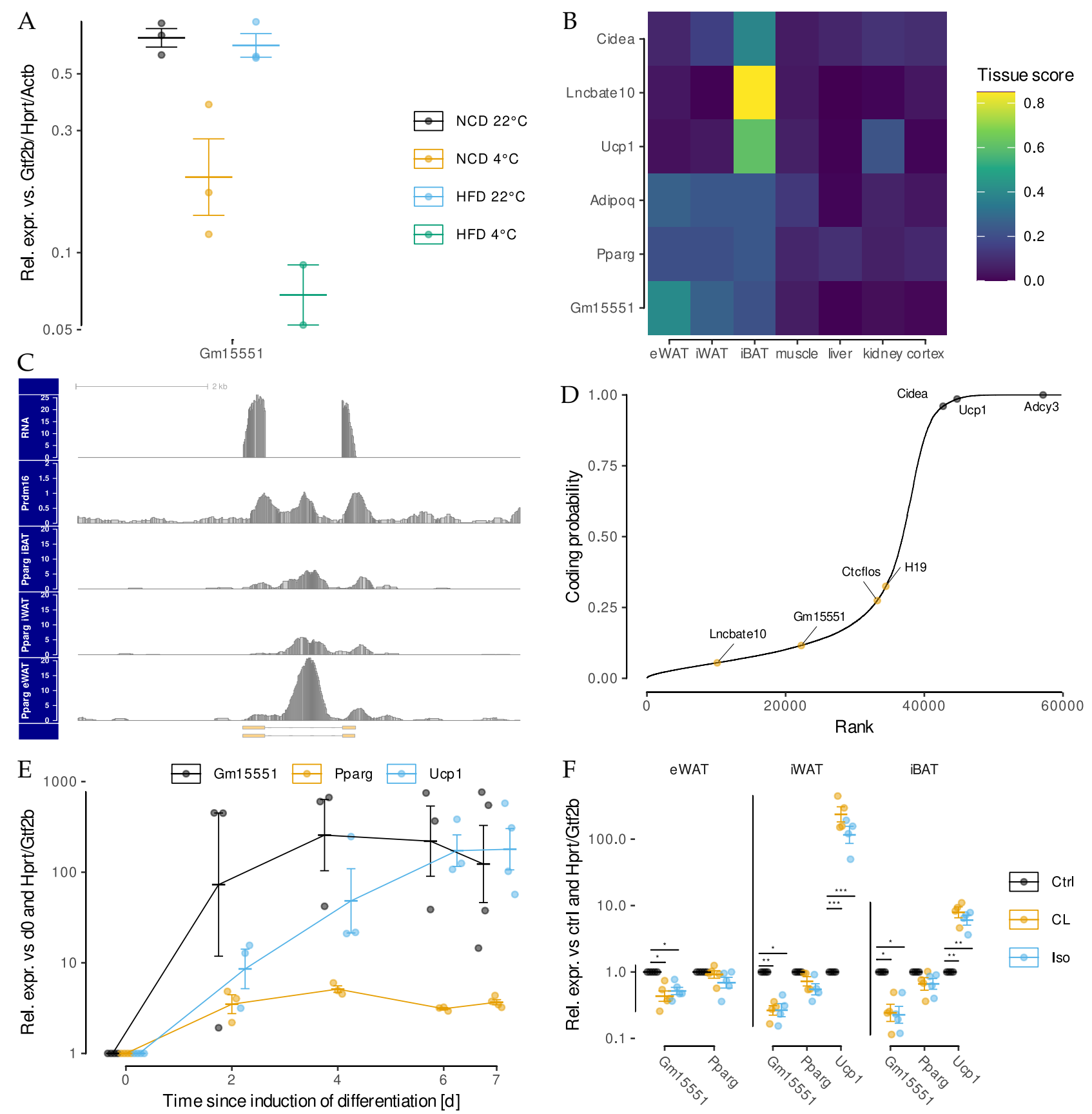

Figure 2: Gm15551 is an adipose tissue specific, diet and temperature regulated lncRNA. 
A
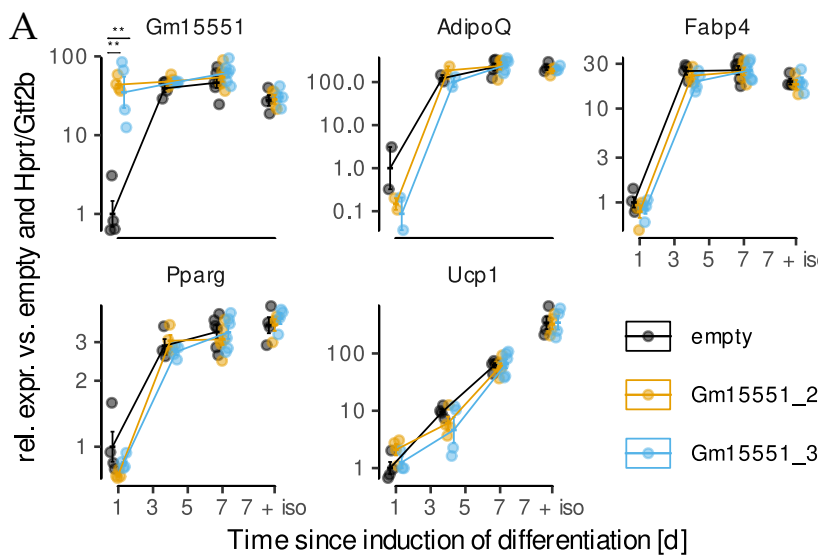

$\theta$ empty

$\because$ Gm15551_2

$\rightarrow$ Gm15551_3

Time since induction of differentiation [d]

C
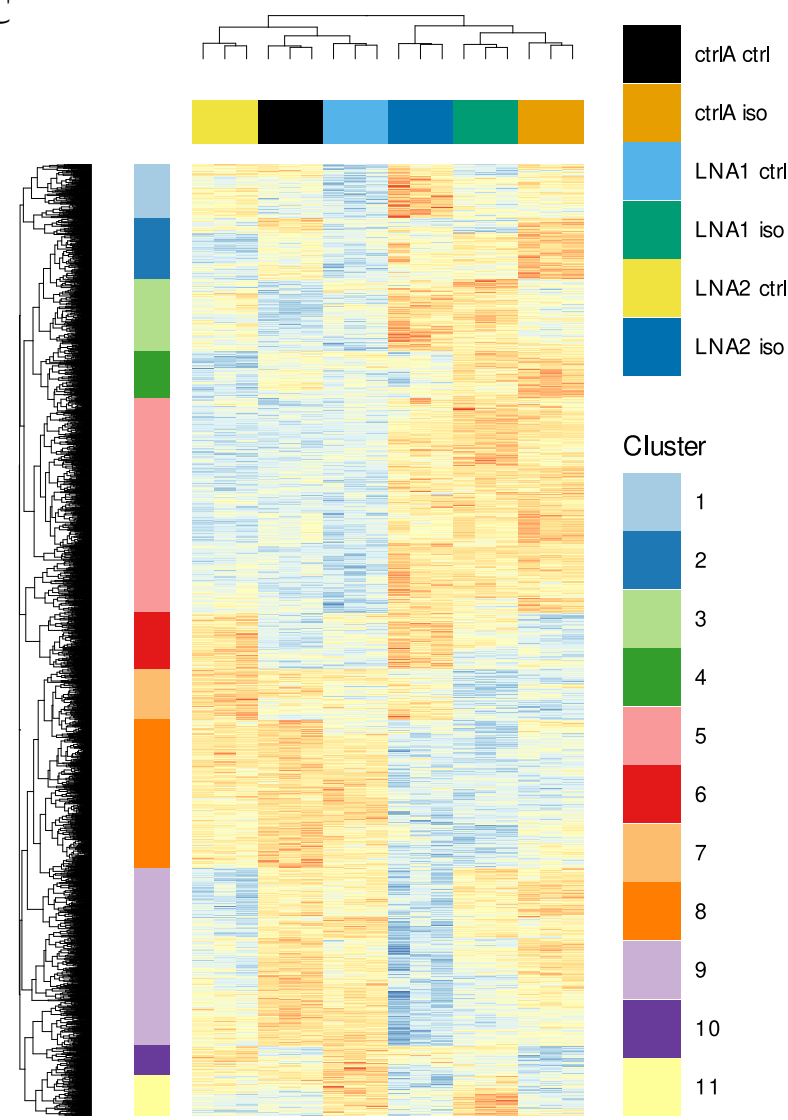

E 4.7 Chrna10 ENSMUSG00000063885

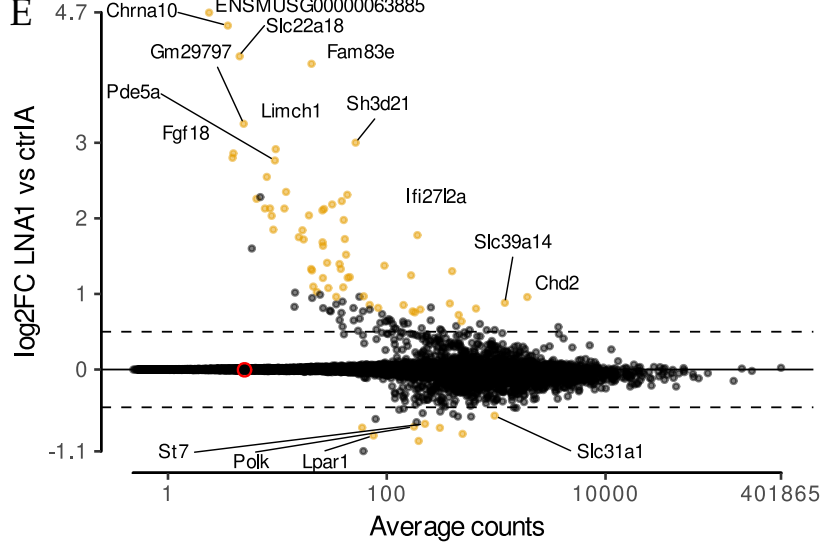

B

d4

d7 Ctrl

d7 Iso

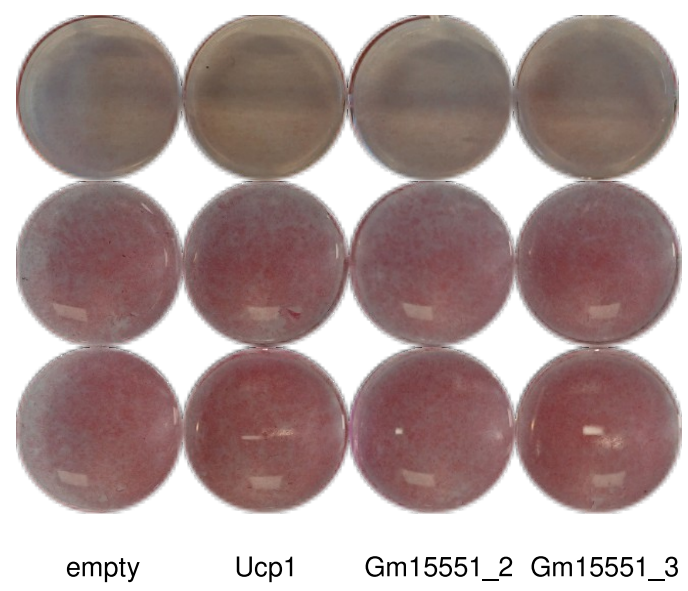

D
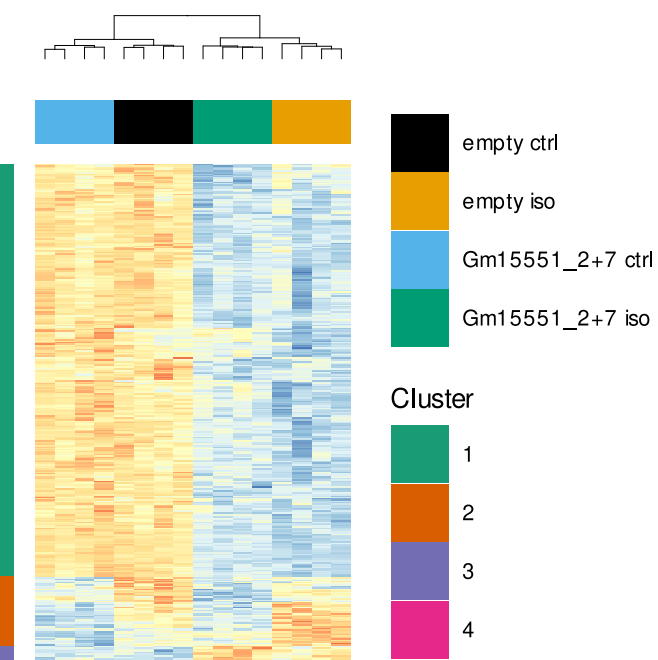

z

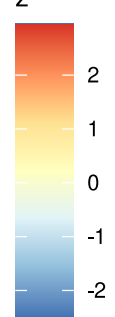

$\mathrm{F}$

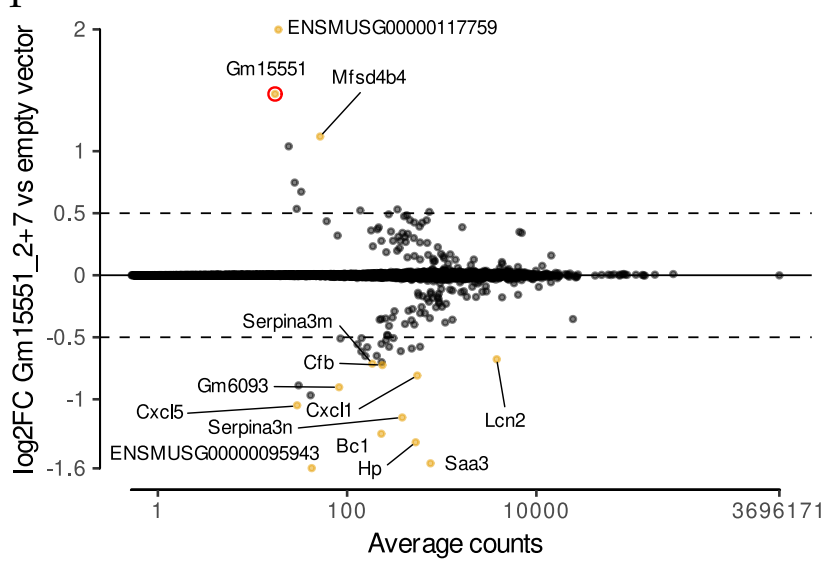

Figure 3: Gm15551 is dispensable for iBAT function in vitro. 
A

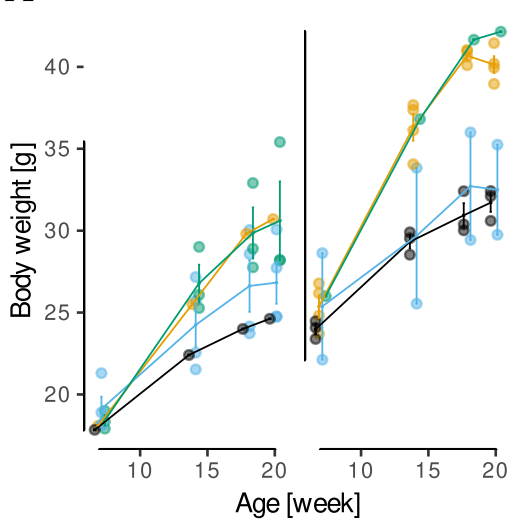

$\underline{\mathrm{C}}$

C

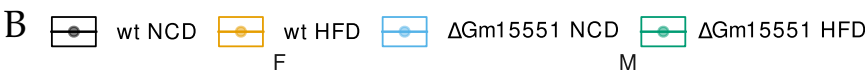

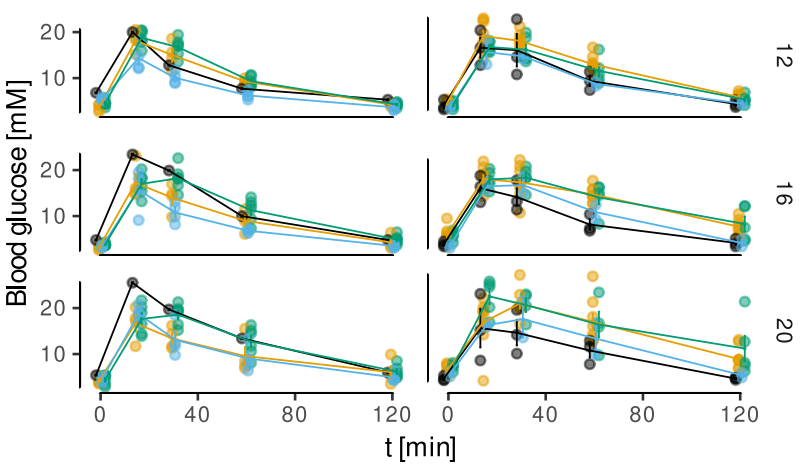

$\frac{\mathrm{D}}{w t N C D}$ - wt HFD $-\triangle \mathrm{Gm1} 15551 \mathrm{NCD}-\triangle \mathrm{Gm} 15551 \mathrm{HFD}$

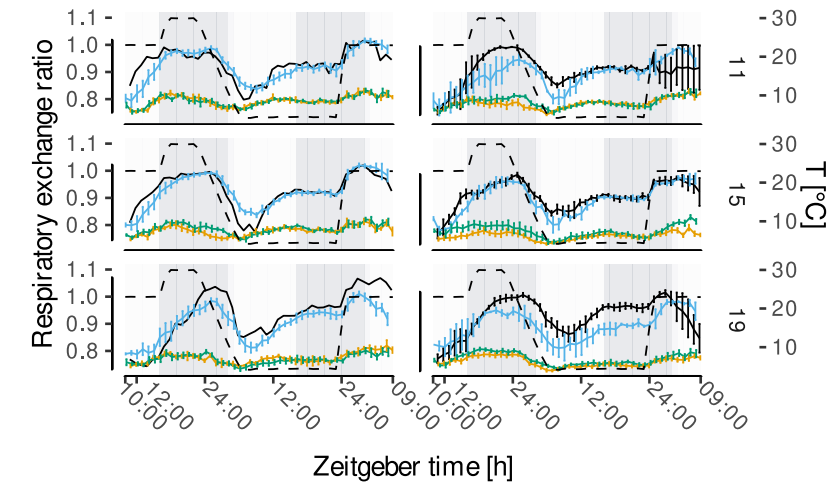

$\mathrm{F}$
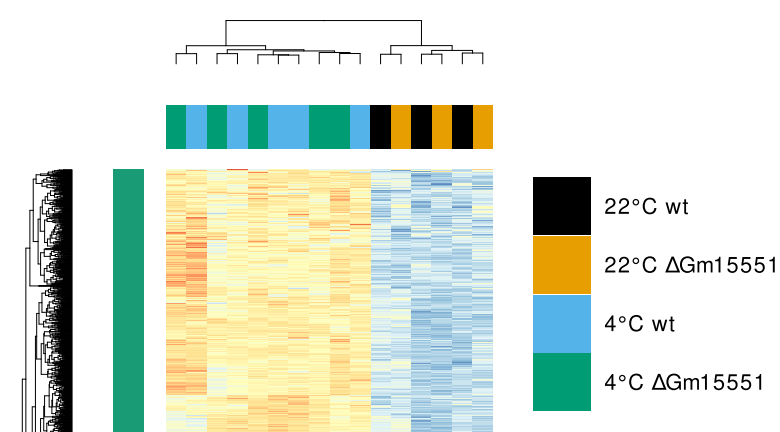

Cluster

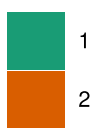

z

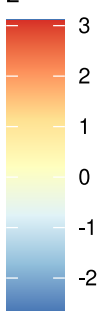

$\mathrm{E}$
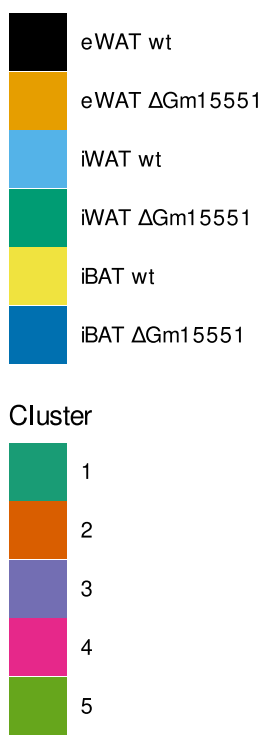

Z

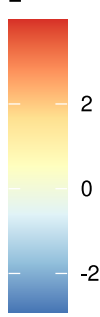

Figure 4: Gm15551 is dispensable for iBAT function in vivo. 


\section{Supplementary figures}

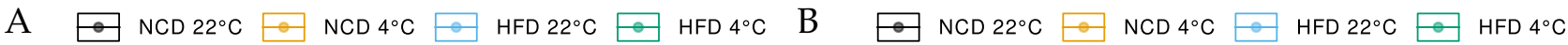
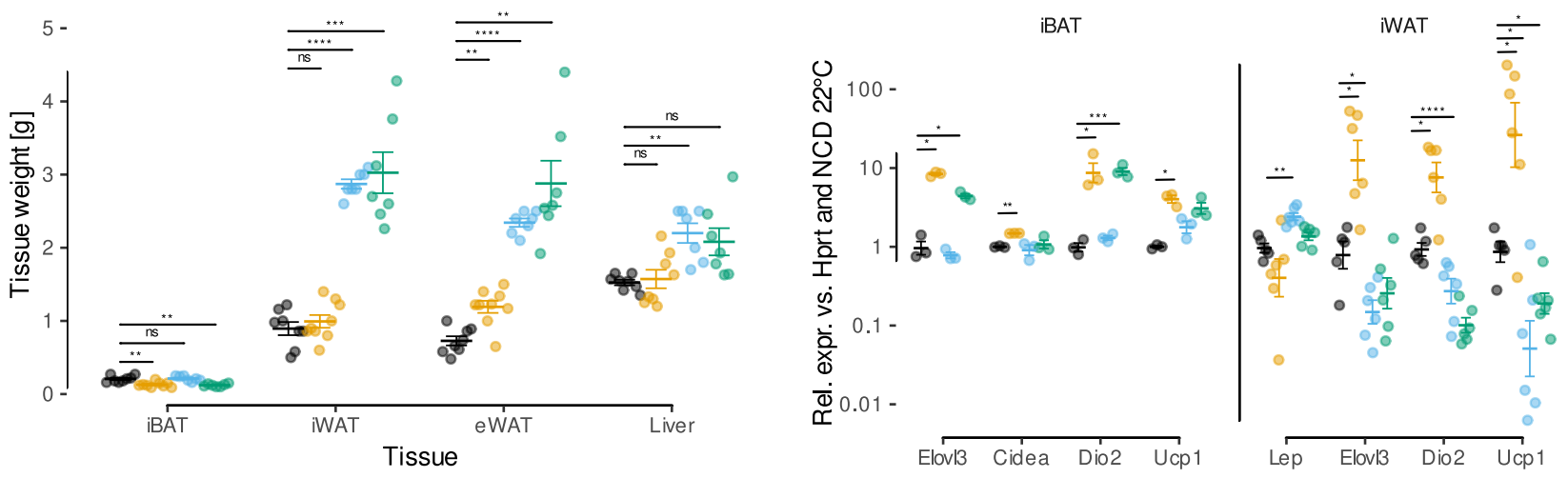

C
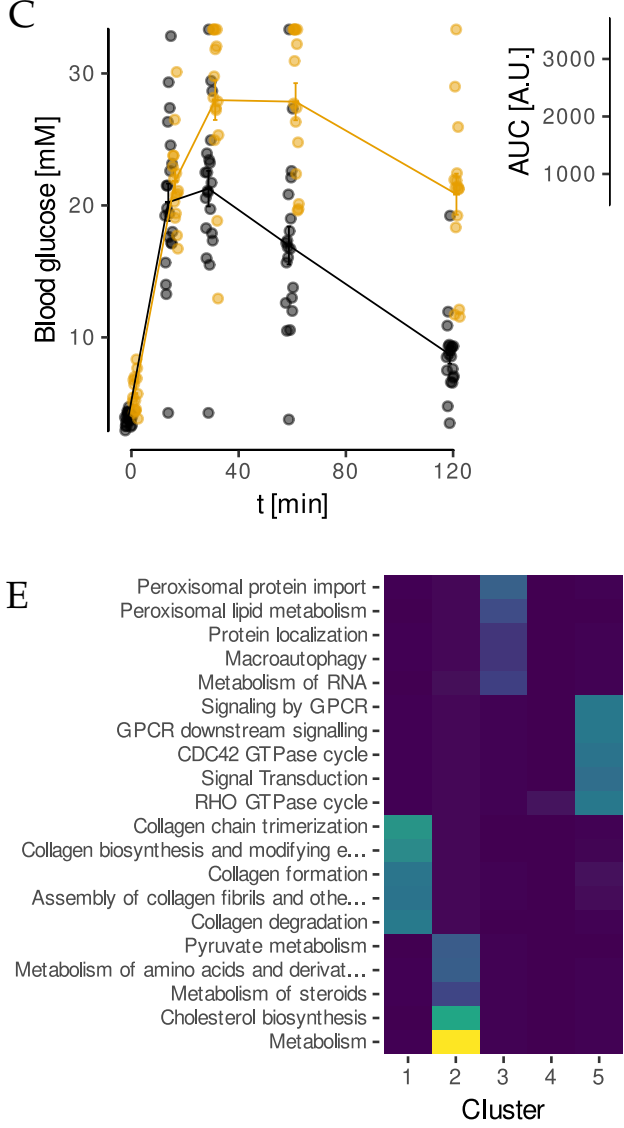

G

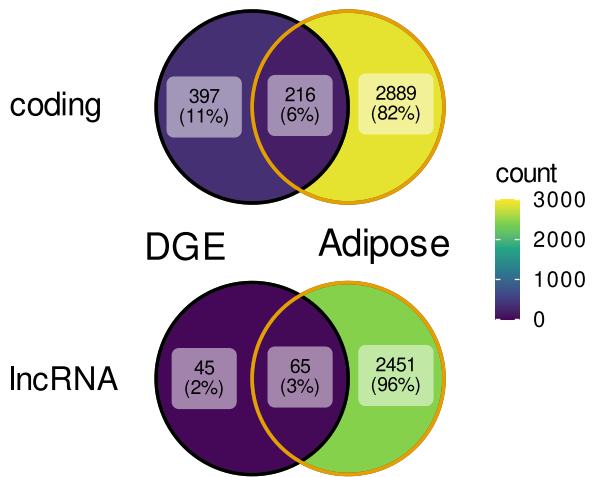

$\mathrm{D} \quad \because \mathrm{NCD} 22^{\circ} \mathrm{C} \boxminus \mathrm{NCD} 4^{\circ} \mathrm{C} \boxminus \mathrm{HFD} 22^{\circ} \mathrm{C} \boxminus \mathrm{HFD} 4^{\circ} \mathrm{C}$
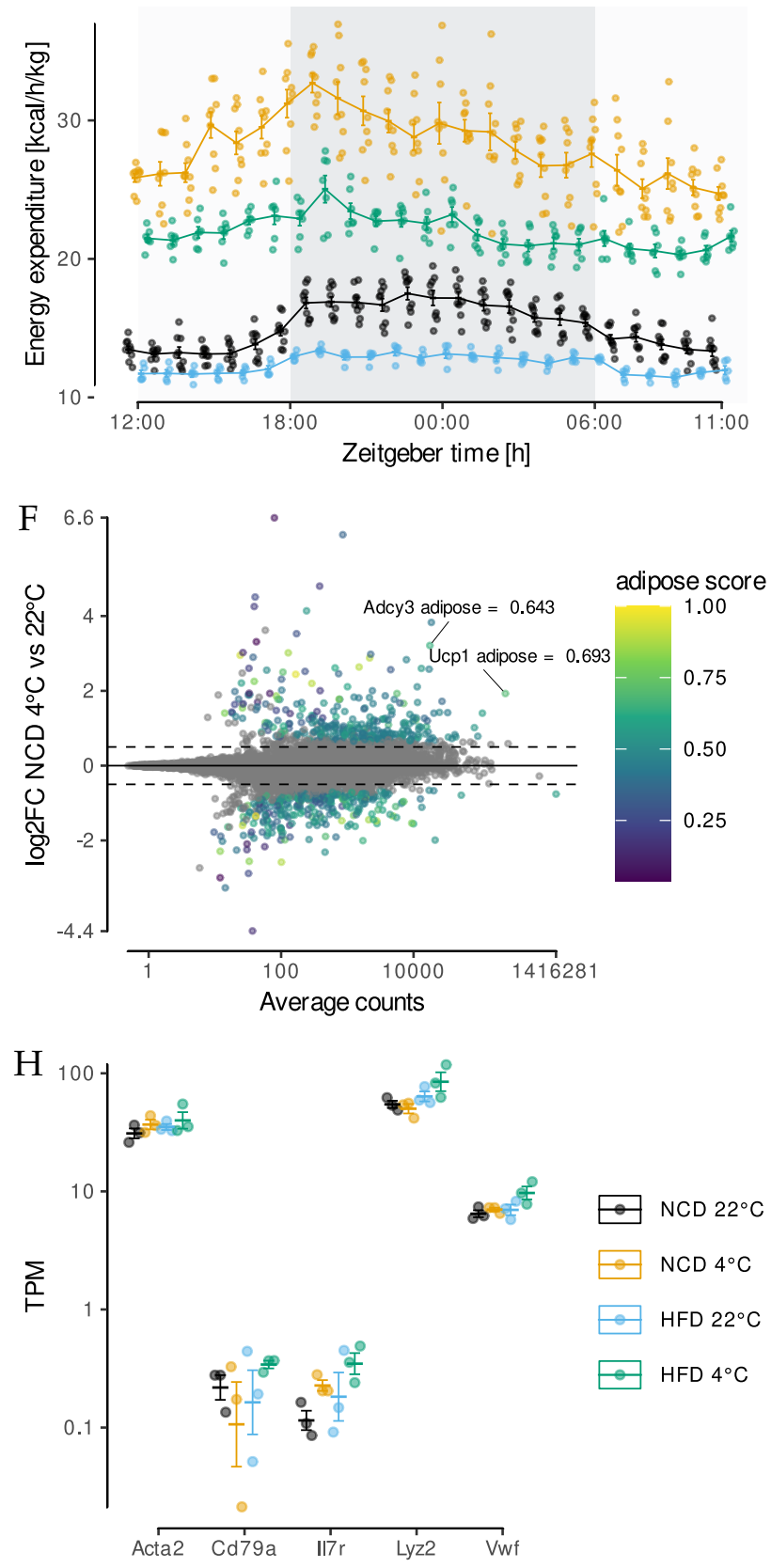

Figure S1: RNA-Seq reveals temperature and obesity dependent changes in iBAT lncRNA expression. 

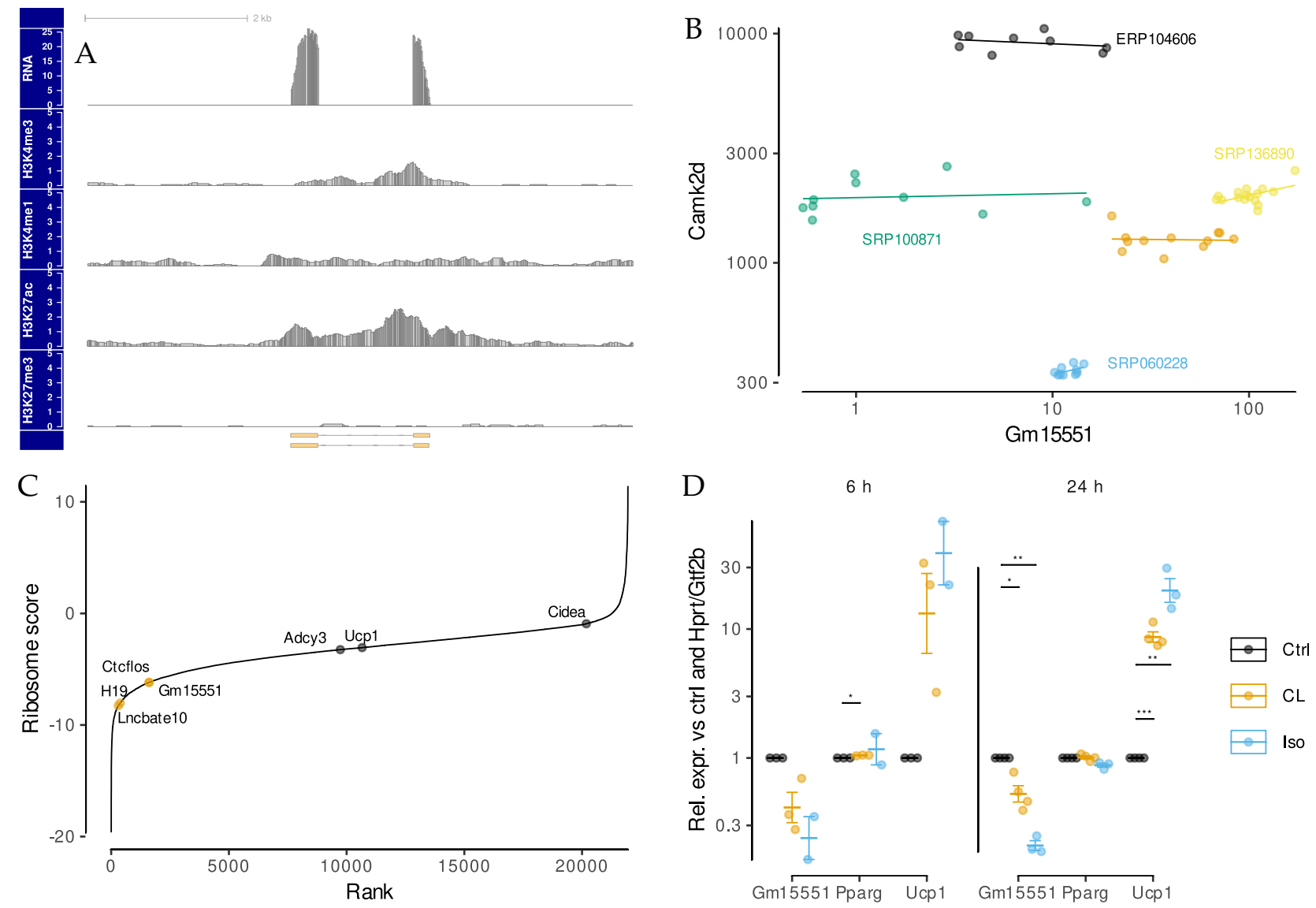

Figure S2: Gm15551 is an adipose tissue specific, diet and temperature regulated lncRNA 

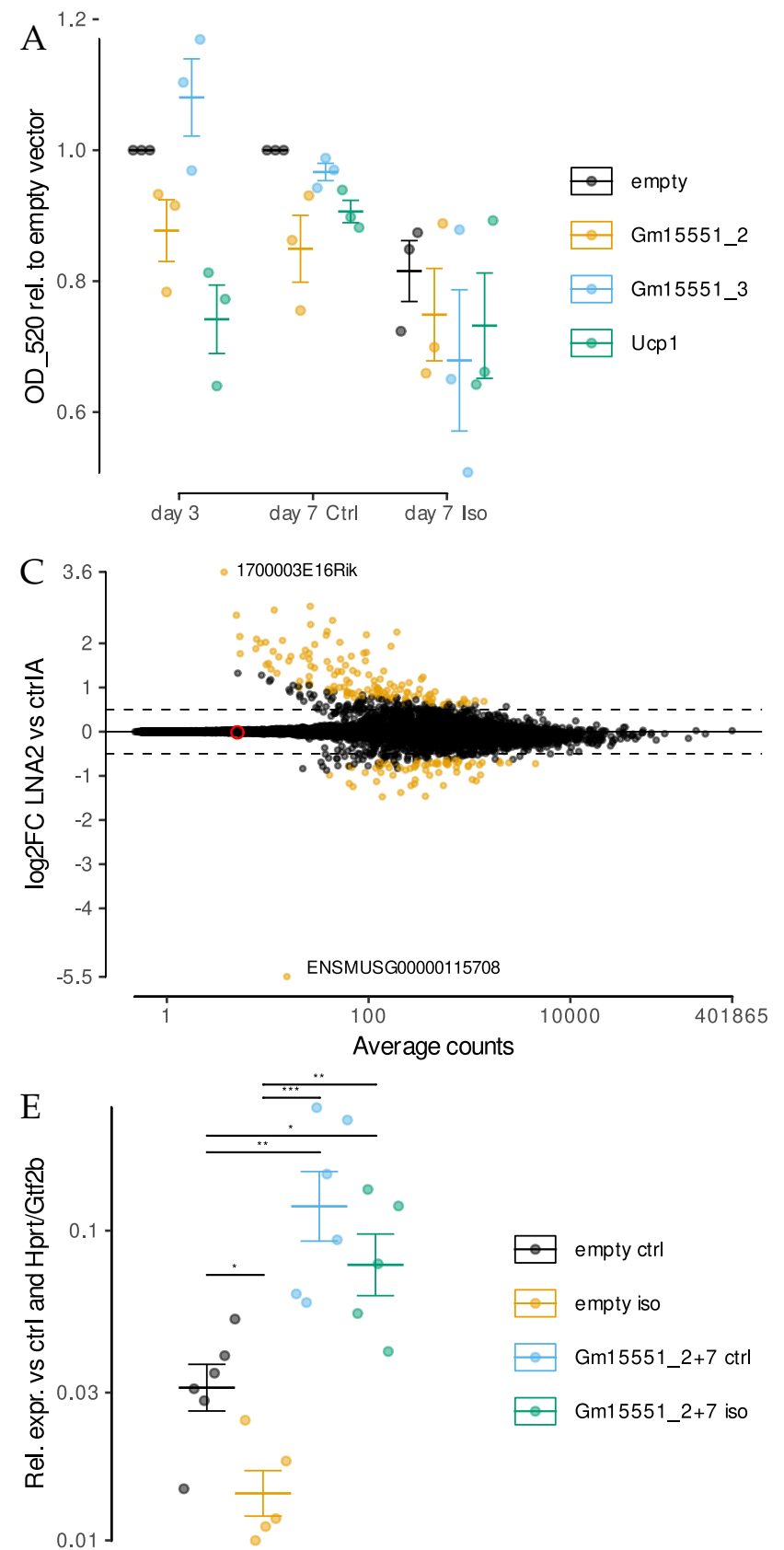

Gm1'5551

G

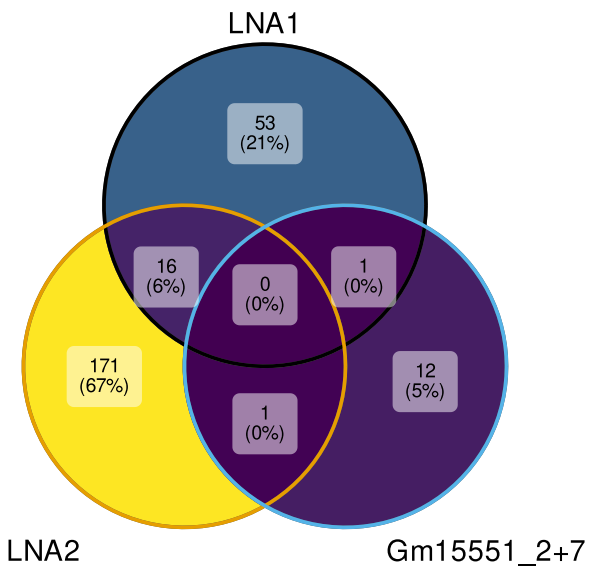

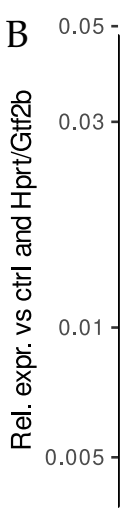

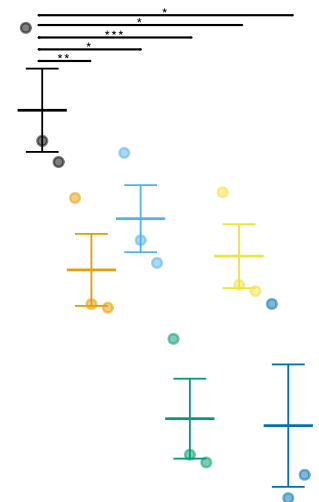

Ctrl_A ctrl

Ctrı_A iso

LNA1 ctrl

LNA1 iso

LNA2 ctrl

LNA2 iso

Gm15 551

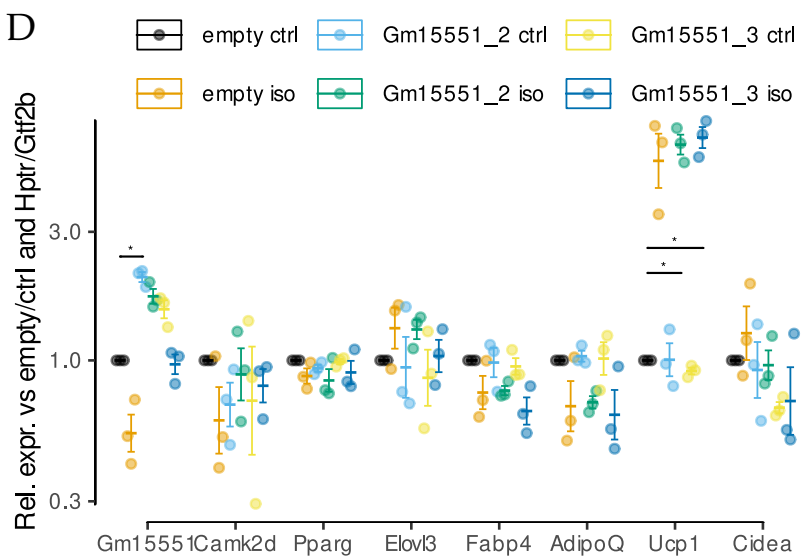

F
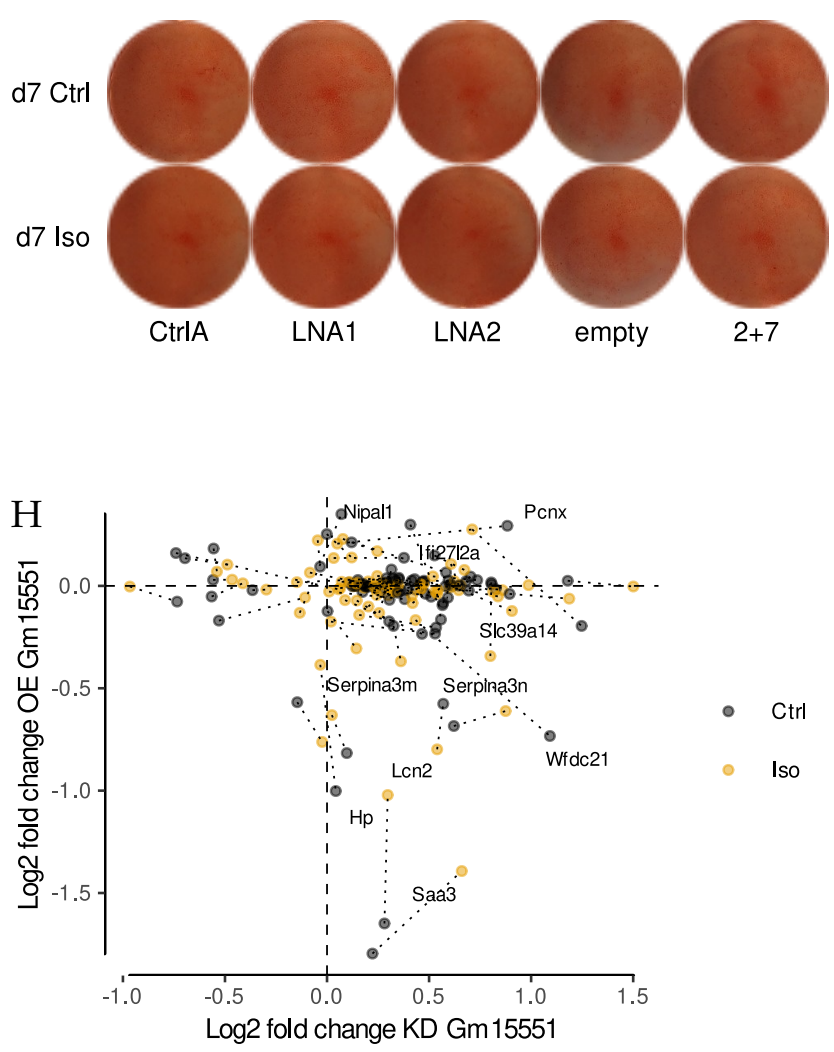

Figure S3: Gm15551 is dispensable for iBAT function in vitro. 
A

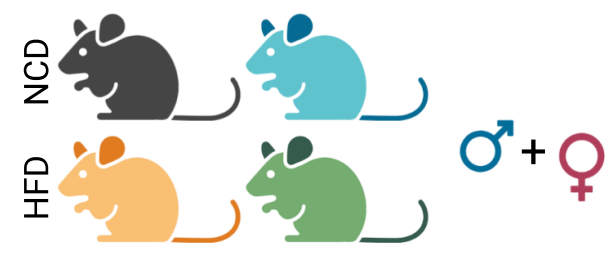

start

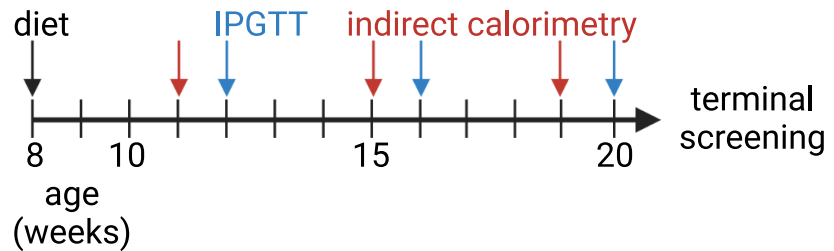

C

wt

$\Delta \mathrm{Gm} 15551$
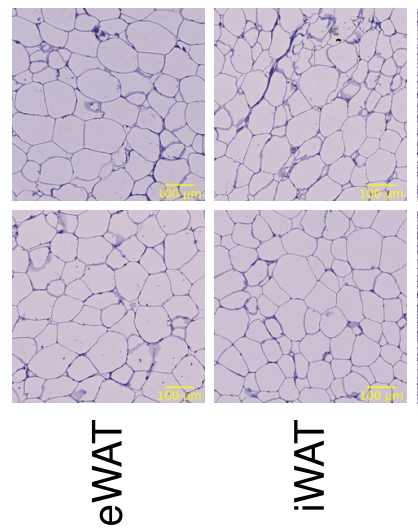

$\mathrm{E}$

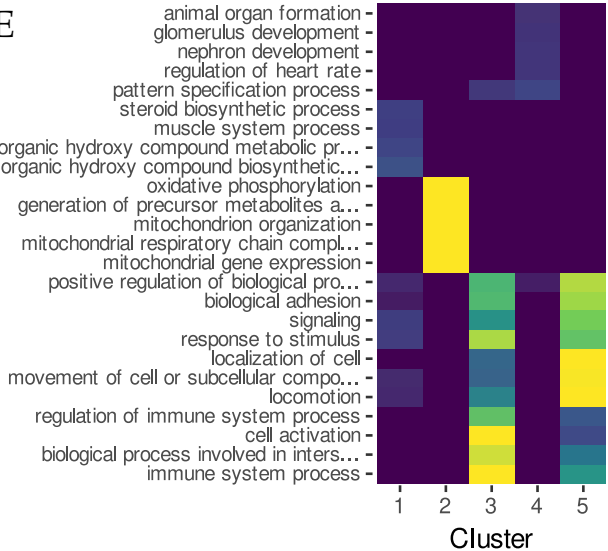

G

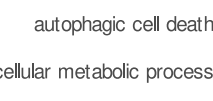

enzyme linked receptor protein signal..

cellular response to stress -

metabolic process -

small molecule metabolic process -

organic acid metabolic process -

cellular lipid metabolic process -

lipid metabolic process -

localization -
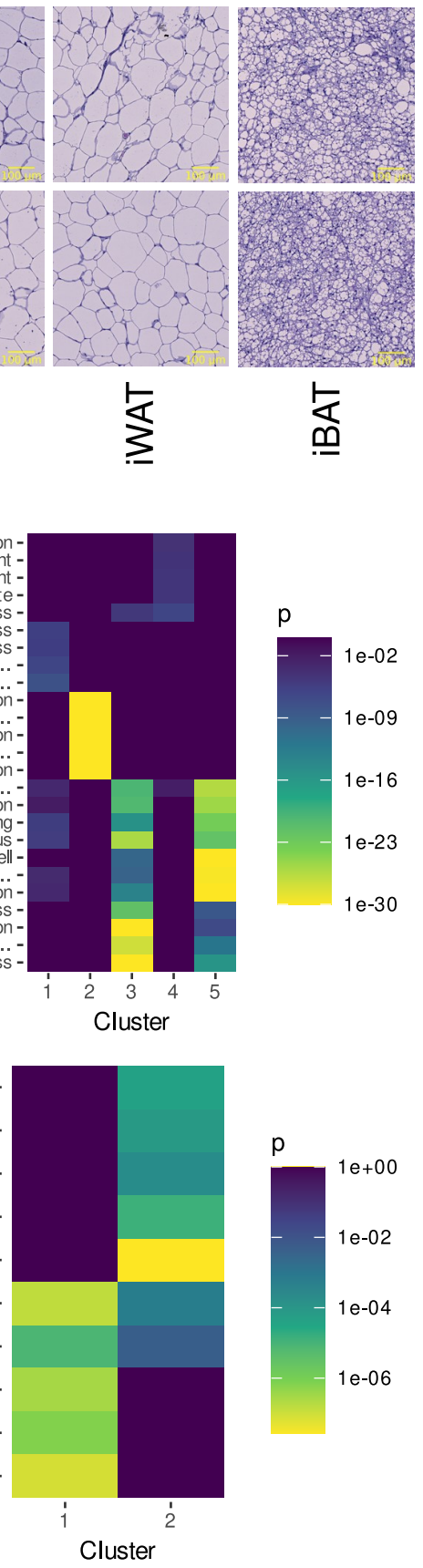

客

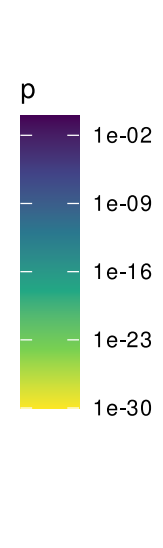

B

$\mathrm{D}$

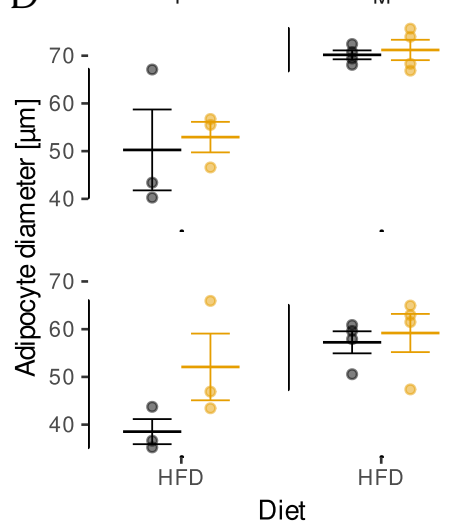

$\sum_{-1}^{\infty}$
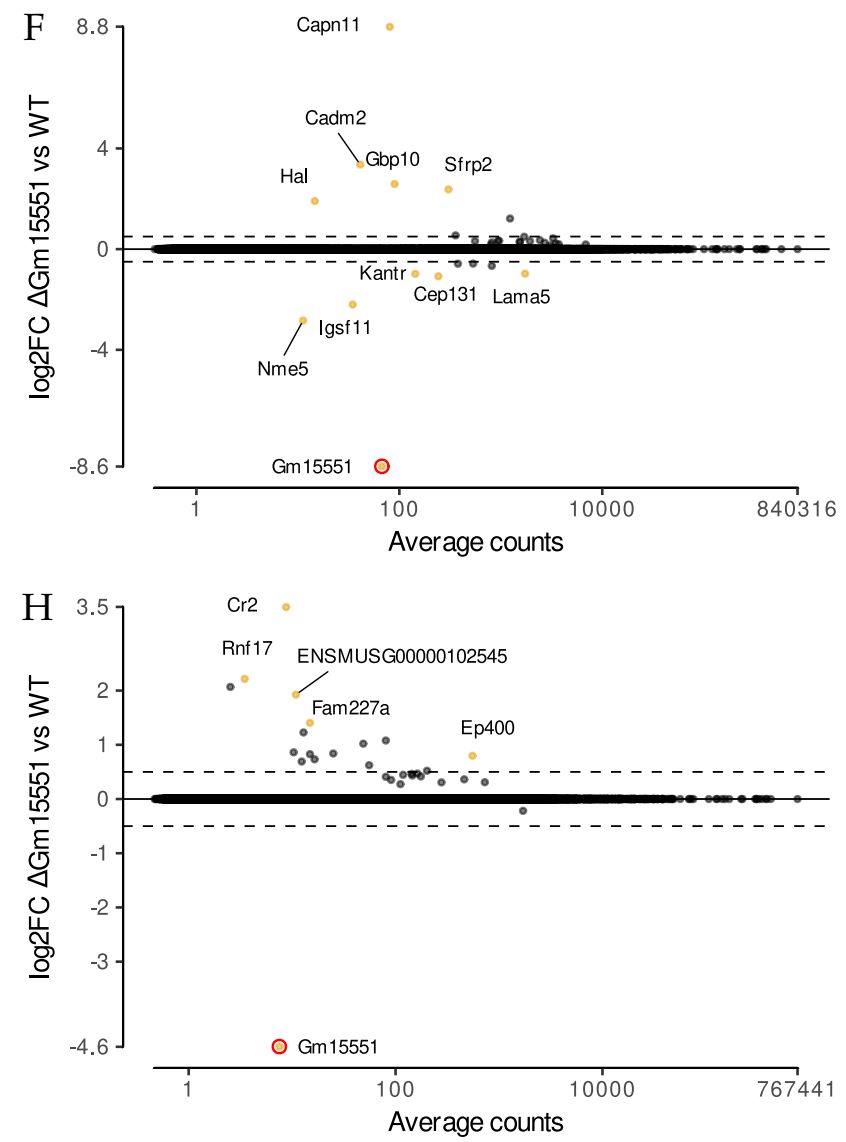

Figure S4: Gm15551 is dispensable for iBAT function in vivo. 


\section{Supplementary tables}

Table S1: Sequences of sgRNAs.

\begin{tabular}{ll}
\hline Target & Sequence \\
\hline Gm15551-2 & CACTTCCAGTTATATAAGCG \\
Gm15551-3 & ACTTCCAGTTATATAAGCGT \\
Gm15551-7 & AGGGTTTTTTGCTAAAACGG \\
Ucp1 & GGGAGTGACGCGCGGCTGGG \\
\hline
\end{tabular}

Table S2: Sequences of qPCR primers.

\begin{tabular}{lll}
\hline Target & forward & reverse \\
\hline Gtf2b & GTGGGATCTGAATGGAGAACTT & CCTGTACCCTTGCCAATCAT \\
Gm15551 & ACGGCGTTGGAAGGCTCT & CACCCGTGCAACGCCTG \\
Ucp1 & CCTTCCCGCTGGACACTG & GGCCTTCACCTTGGATCTGA \\
Pparg & CACAATGCCATCAGGTTTGG & CAGCTTCTCCTTCTCGGCCT \\
AdipoQ & GACACCAAAAGGGCTCAGG & TTAGGACCAAGAAGACCTGC \\
Fabp4 & AAATCACCGCAGACGACAGG & CGCCATCTAGGGTTATGATGCT \\
Elovl3 & AGCAAGGTTGTTGAACTGGGA & GACGCTTACGCAGGATGATGA \\
Cidea & AGGCCGTGTTAAGGAATCTGCT & GCCCAGTACTCGGAGCATGT \\
Lep & TGTGCTGCAGATAGCCAATGA & AGATGGAGGAGGTCTCGGAGA \\
\hline
\end{tabular}

Table S3: Antibodies used for chromatin modification ChIP-Seq.

\begin{tabular}{llr}
\hline Target & Source & Dilution \\
\hline Histone H3K27ac & Active Motif 39133 & $5 \mu \mathrm{l}$ \\
Histone H3K27me3 & Active Motif 39155 & $5 \mu \mathrm{l}$ \\
Histone H3K4me1 & Active Motif 39297 & $10 \mu \mathrm{l}$ \\
Histone H3K4me3 & Active Motif 39159 & $3 \mu \mathrm{l}$ \\
\hline
\end{tabular}

\section{Legends}

\section{Fig 1: RNA-Seq reveals temperature and obesity dependent changes in iBAT IncRNA expression}

A Experimental design. Total transcriptomes from the iBAT of 20 week old mice housed at $22^{\circ} \mathrm{C}$ or $4{ }^{\circ} \mathrm{C}$ for $24 \mathrm{~h}(\mathrm{n}=3)$ fed either a high fat or a control diet for 12 weeks were analysed together with the total-transcriptomes from seven metabolically active tissues ( $n=1$, GSE121345). The union of GENCODE and RNAcentral annotated genes was used for the analysis to reveal lncRNAs which are both adipose tissue specific and regulated by physiologically relevant stimuli. Created with BioRender.com. B Hierarchical clustering of genes differentially regulated by diet and cold treatment in adipose tissue (likelihood ratio test, FDR $<0.001$ ). Colour code depicts row wise standardised expression. C GO enrichment analysis for the gene clusters shown in B. D Expression levels and changes for lncRNA genes in iBAT from cold treated compared to control mice on control diet. Genes 
showing significant differential gene expression are colour coded indicating their adipose tissue specificity (wald test, $\log 2$ fold change $(\log 2 \mathrm{FC})>0.5, \mathrm{n}=6, s<0.05)$.

\section{Fig S1: RNA-Seq reveals temperature and obesity dependent changes in iBAT IncRNA expression}

A Adipose tissues and liver weights of 20 week old mice after cold and/or HFD treatment ( $t$-test, $\mathrm{n}=7$ - 9). B Expression of common and brown specific adipose marker genes and the macrophage marker Emr1 in iBAT and iWAT of cold and/or HFD challenged mice ( $t$-test, $\mathrm{n}=3-6)$. C IPGTT of HFD and control diet fed animals at 12 weeks to 14 weeks of age ( $t$-test, $\mathrm{n}=15)$. $\mathbf{D}$ Energy expenditure of HFD or control animals kept at either $22{ }^{\circ} \mathrm{C}$ or $4{ }^{\circ} \mathrm{C}$ measured by indirect calorimetry $(\mathrm{n}=5-8)$. E Reactome pathway enrichment analysis for the clusters in Fig1 A. F Expression levels and changes for coding genes in iBAT from cold treated compared to control mice. Genes showing significant differential gene expression are colour coded indicating their adipose tissue specificity (wald test, $\mathrm{n}$ $=6, s<0.05, H_{0}: \log 2 \mathrm{FC}>0.5$ ). G Overlap of differential gene expression (wald test, $s<0.05$ ) and adipose tissue specificity (adipose score $>0.5$ ) for coding and lncRNA genes. $\mathbf{H}$ Expression levels of immune cell marker genes in the total transcriptomes from iBAT of cold and/or HFD treated mice.

\section{Fig 2: Gm15551 is an adipose tissue specific, diet and temperature regulated IncRNA}

A Expression of Gm15551 in iBAT from cold treated and/or HFD fed mice. B Expression profile of Gm15551, the common adipocyte marker genes Pparg and Adipoq as well as the iBAT specific lncRNA Lncbate10 and the brown adipocyte marker genes Ucp1 and Cidea in seven metabolically active tissues. C Genomic locus of Gm15551 showing the RNA expression as well as binding sites of Prdm16 in iBAT (PRJNA269620) and Pparg in eWAT, iWAT and iBAT (PRJNA177164). D Ranked coding probability of all genes expressed in the dataset as calculated by CPAT. Indicated are Gm15551, the coding genes Ucp1, Adcy3 and Cidea as well as the lncRNAs Ctcflos, H19 and LncBate10. E, F Expression profiles of Gm15551, the common adipocyte marker gene Pparg and the brown adipocyte marker gene Ucp 1 during the differentiation of PIBA cells (E) and in fully differentiated primary adipocytes (F) stimulated for $24 \mathrm{~h}$ with the non-selective $\beta$-adrenergic agonist isoproterenol or the $\beta_{3}$-specific agonist CL316243 (paired $t$-test, $\mathrm{n}=4-5$ ).

\section{Fig S2: $\mathrm{Gm} 15551$ is an adipose tissue specific, diet and temperature regulated IncRNA}

A Genomic locus of Gm15551 showing the RNA expression as well as chromatin modifications in iBAT of control mice. B Expression of Gm15551 vs. Camk2d in indicated public RNA-Seq data sets. (Orange is the dataset from this study.) C Ranked ribosome scores as calculated from TRAP-Seq of murine iBAT (PRJNA402074). D Expression of Gm15551, Pparg and Ucp1 in fully differentiated PIBA cells, stimulated with isoproterenol or CL316243 for either $6 \mathrm{~h}$ or $24 \mathrm{~h}$ (paired $t$-test, $\mathrm{n}=2-3$ ).

\section{Fig 3: Gm15551 is dispensable for iBAT function in vitro}

A, B Gene expression profiles (A) of Gm15551, the common adipocyte marker genes Pparg, Adipoq and Fabp 4 as well as the brown adipocyte marker gene Ucp1 (paired $t$-test, $\mathrm{n}=2-6$ ) and oil red o staining (F) of wt1-SAM cells transfected with plasmids coding for sgRNAs targeting Gm15551, Ucp1 or empty vector two days before induction of differentiation. C, D Hierarchical clustering of genes 
differentially regulated by $\beta$-adrenergic stimulation and/or knockdown (C) or overexpression (D) in mature adipocytes at day 4 of differentiation (LRT, $\mathrm{n}=3$ (C) or 4 (D), $p<0.001$ ). E, F Effect of knockdown (E) or overexpression (F) of Gm15551 on gene expression in mature wt1-SAM cells. The $\log 2 \mathrm{FC}$ is the average over the effect in isoproterenol stimulated and control cells (wald test, $\log 2 \mathrm{FC}$ $>0.5, \mathrm{n}=6(\mathrm{E})$ or $8(\mathrm{~F}), s<0.05)$.

\section{Fig S3: Gm15551 is dispensable for iBAT function in vitro}

A Quantification of lipid accumulation by oil red O staining of wt1-SAM cells transfected with plasmids encoding sgRNAs targeting Gm15551 or empty vector two days before induction of differentiation (paired $t$-test, $\mathrm{n}=3$ - 6). B Efficiency of the knockdown of Gm15551 using wo different LNAs in mature wt1-SAM cells (paired $t$-test, $\mathrm{n}=3$ ). C Effect of knockdown of Gm15551 using LNA2 at day 4 of differentiation on gene expression in mature wt1-SAM cells (wald test, $\log 2 \mathrm{FC}>0.5, \mathrm{n}=$ 6, s < 0.05). D Gene expression of Gm15551, Camk2d, the general adipocyte markers Pparg, Elovl3, Adipoq and Fabp4 as well as the brown adipocyte marker genes Ucp1 and Cidea in mature wt1-SAM cells after overexpression of Gm15551 at day 4 of differentiation (paired $t$-test, $\mathrm{n}=3$ ). E Efficiency of overexpression of Gm15551 in mature wt1-SAM cells using a combination of 2 sgRNAs (wald test, $\log 2 \mathrm{FC}>0.5, \mathrm{n}=6, s<0.05)$. F Oil red O staining of mature wt1-SAM cells after transfection with LNAs or plasmids encoding sgRNAs targeting Gm15551 at day 4 of differentiation. F Effect of gain and loss of function of Gm15551 in mature adipocytes on lipid accumulation. G, H Overlap between genes showing differential regulation and comparison of their gene expression changes in wt1-SAM cells upon knockdown or overexpression of Gm15551 $(s<0.05)$ at day 4 of differentiation.

\section{Fig 4: Gm15551 is dispensable for iBAT function in vivo}

A Body weight of $\Delta \mathrm{Gm} 15551$ and wild type mice fed a high fat or control diet. B IPGTT of 12, 16 and 20 week old $\Delta \mathrm{Gm} 15551$ and wild type mice fed a high fat or control diet. C, D Energy expenditure (C) and respiratory exchange rates (D) of 12, 16 and 20 week old $\Delta \mathrm{Gm} 15551$ and wild type mice fed a high fat or control diet. E Hierarchical clustering of genes differentially regulated between adipose tissues or by knockout of Gm15551 in 12 week old mice (LRT, $\mathrm{n}=3, p<0.001$ ). F Hierarchical clustering of genes differentially regulated by temperature or by knockout of Gm15551 in in iBAT of 12 week old mice (LRT, $\mathrm{n}=3$ or $5, p<0.001$ ).

\section{Fig S4: Gm15551 is dispensable for iBAT function in vivo}

A Experimental design. Male and female $\Delta \mathrm{Gm} 15551$ and wild type litter mates were either put on HFD or control diet for 12 weeks starting at 8 weeks of age. IPGTT and indirect calorimetry measurements were repeatedly performed at the indicated timepoints. Created with BioRender.com. B Body fat percentage of $\Delta \mathrm{Gm} 15551$ and wild type mice fed a high fat or control diet. C, D Representative microphotographs (C) and adipocyte diameters (D) in different adipose tissue from 20 week old wt and $\Delta \mathrm{Gm} 15551$ mice fed a HFD. E GO enrichment analysis for the gene clusters shown in Fig $4 \mathrm{E}$. F Gene expression changes induced by the knockout of Gm15551 in different adipose tissues (wald test, $\left.\mathrm{n}=3, s<0.05, H_{0}: \log 2 \mathrm{FC}>0.5\right)$. G GO enrichment analysis for the gene clusters shown in Fig 4 F. H Gene expression changes induced by the knockout of Gm15551 in iBAT of cold treated and room temperature housed mice (wald test, $\mathrm{n}=3$ or $5, s<0.05, H_{0}: \log 2 \mathrm{FC}>0.5$ ). 